\title{
Local Impact of Stochastic Shallow Convection on Clouds and Precipitation in the Tropical Atlantic
}

\author{
Mirjana SakradziJa, ${ }^{\mathrm{a}, \mathrm{b}}$ Fabian SenF, ${ }^{\mathrm{c}}$ LeOnhard Scheck, ${ }^{\mathrm{d}, \mathrm{b}}$ Maike Ahlgrimm, ${ }^{\mathrm{e}}$ AND Daniel KLOCKe ${ }^{\mathrm{e}, \mathrm{b}}$ \\ ${ }^{a}$ Max Planck Institute for Meteorology, Hamburg, Germany \\ ${ }^{\mathrm{b}}$ Hans Ertel Centre for Weather Research, Deutscher Wetterdienst, Offenbach am Main, Germany \\ ${ }^{\mathrm{c}}$ Leibniz-Institut für Troposphärenforschung, Leipzig, Germany \\ ${ }^{\mathrm{d}}$ Ludwig-Maximilian-Universität, Munich, Germany \\ e Deutscher Wetterdienst, Offenbach am Main, Germany
}

(Manuscript received 7 April 2020, in final form 6 October 2020)

\begin{abstract}
The local impact of stochastic shallow convection on clouds and precipitation is tested in a case study over the tropical Atlantic on 20 December 2013 using the Icosahedral Nonhydrostatic Model (ICON). ICON is used at a grid resolution of $2.5 \mathrm{~km}$ and is tested in several configurations that differ in their treatment of shallow convection. A stochastic shallow convection scheme is compared to the operational deterministic scheme and a case with no representation of shallow convection. The model is evaluated by comparing synthetically generated irradiance data for both visible and infrared wavelengths against actual satellite observations. The experimental approach is designed to distinguish the local effects of parameterized shallow convection (or lack thereof) within the trades versus the ITCZ. The stochastic cases prove to be superior in reproducing low-level cloud cover, deep convection, and its organization, as well as the distribution of precipitation in the tropical Atlantic ITCZ. In these cases, convective heating in the subcloud layer is substantial, and boundary layer depth is increased as a result of the heating, while evaporation is enhanced at the expense of sensible heat flux at the ocean's surface. The stochastic case where subgrid shallow convection is deactivated below the resolved deep updrafts indicates that local boundary layer convection is crucial for a better representation of deep convection. Based on these results, our study points to a necessity to further develop parameterizations of shallow convection for use at the convection-permitting resolutions and to assuredly include them in weather and climate models even as their imperfect versions.
\end{abstract}

KEYWORDS: Intertropical convergence zone; Convective clouds; Precipitation; Cloud resolving models; Convective parameterization; Stochastic models

\section{Introduction}

At horizontal resolutions of weather and climate models of one to several kilometers, deep convection is usually treated explicitly instead of being fully or partially parameterized. This approach alleviates some of the uncertainties in the representation of deep convective systems and their coupling to the resolved dynamics, which brings significant improvements to modeling of the convective diurnal cycle and precipitation (see the review of Prein et al. 2015, and references therein). Though the improvement is evident compared to model resolutions on the order of $10 \mathrm{~km}$, the deep convective updrafts are still underresolved and some biases in the representation of clouds and precipitation are still present at such convection-permitting (CP) scales (Roberts and Lean 2008; Baldauf et al. 2011; Langhans et al. 2013; Fosser et al. 2015; Brisson et al. 2016; Liu et al. 2017; Hentgen et al. 2019). In this study, we argue that the bias in the distribution of clouds and precipitation in a CP model largely originates from the misrepresentation of the local shallow convection.

Misrepresentation of shallow convection at $\mathrm{CP}$ resolutions can occur in at least two different ways. The first is to simply neglect the need for a parameterization, while the second is to adopt a scheme originally developed for coarser-resolution

Corresponding author: Mirjana Sakradzija, mirjana.sakradzija@ mpimet.mpg.de models, without making necessary adjustments. In a study where no subgrid convection scheme is used, the position and width of ITCZ depend on horizontal resolution of the model (Hohenegger et al. 2020), which indicates a high relevance of smallest-resolved convective scales for the ITCZ region and existing biases of precipitation. In their study, the net shortwave radiation systematically increases with the increase of model resolution from 80 to $2.5 \mathrm{~km}$ because of reductions in the amount of low-level clouds over the subtropical oceans. However, the cloud amount is still largely overestimated at the $2.5 \mathrm{~km}$ resolution when the parameterization of convection is not used (Senf et al. 2018, 2020, see also section 3 of the present study). On the other hand, the models that include a parameterization of shallow convection suffer from biases and uncertainties due to inadequate representation of shallow clouds at the subgrid scale (Nam et al. 2012). The shallow convection schemes cause biases even at coarse resolutions for which they were initially developed, and thus their direct application at the $\mathrm{CP}$ resolutions further degrades their performance. This could be one reason why the state-of-the-art parameterizations are still misrepresenting shallow convective processes and have repercussions for the shortwave radiation budget (PedruzoBagazgoitia et al. 2019). The outgoing longwave and net shortwave fluxes at the top of the atmosphere have substantial biases compared to satellite data at a resolution of about $2 \mathrm{~km}$, which is largely attributable to the misrepresentation of clouds (Hentgen et al. 2019; Senf et al. 2020). 
a) SEVIRI

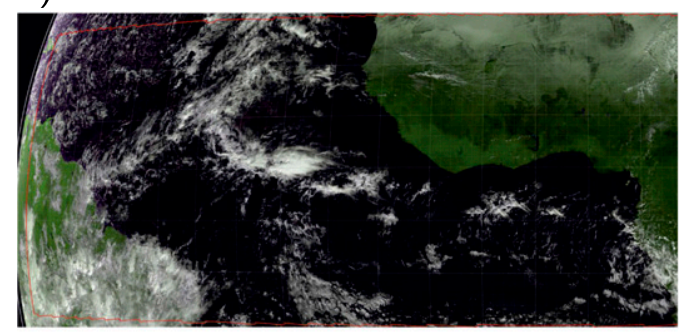

c) D-OPER

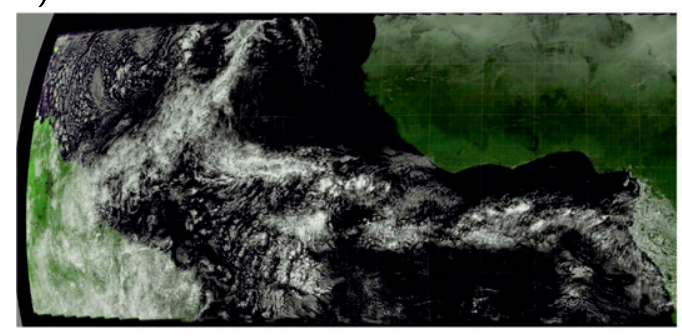

b) NOCONV

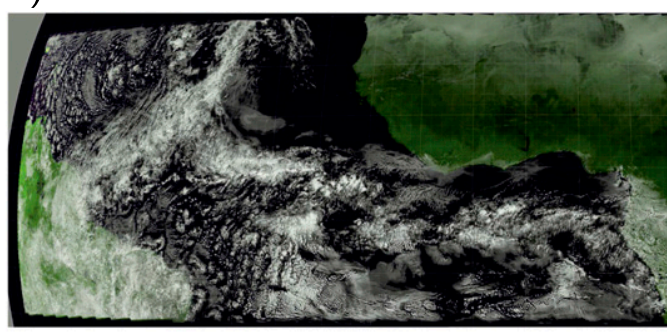

d) S-NOMFL

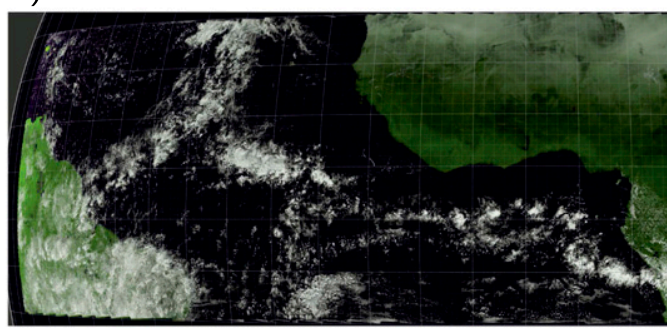

FIG. 1. (a) Reflectance based on the SEVIRI data at 1200 UTC compared to the synthetic reflectance derived using the ICON model (b) without subgrid convection, (c) with operational T-B shallow convection, and (d) with the stochastic shallow convection scheme. In these RGB color images the $0.6 \mu \mathrm{m}$ reflectance is used for the red, the $0.8 \mu \mathrm{m}$ reflectance is used for the green, and the mean of the 0.6 and $0.8 \mu \mathrm{m}$ reflectances is used for the blue component.

The possible importance of shallow convection for the largescale circulation and ITCZ has previously been suggested to arise through modulation of evaporation in the subtropics via changes in low-level vertical transports of moisture by shallow convection and lateral transport of moisture toward the ITCZ (Tiedtke et al. 1988; Slingo et al. 1994; Neggers et al. 2006). However, these studies involved coarse resolutions and parameterized deep convection. The importance of subgrid shallow convection for the Hadley cell and the Atlantic ITCZ has not yet been assessed in the CP models. More importantly for our study, the impact of local shallow convection on the tropical clouds and ITCZ, isolated from the remote impact through the large-scale circulation, has not been studied before.

A representation of shallow convection aimed specifically at the CP resolutions was developed by Sakradzija et al. (2015) and implemented in the ICOsahedral Nonhydrostatic (ICON) model by Sakradzija et al. (2016) and Sakradzija and Klocke (2018). This parameterization includes a stochastic routine that resamples the cloud-base mass-flux, introduces convective memory and reconstructs a resolution-dependent distribution of the mass flux to correct the closure of the underlying Tiedtke-Bechtold (T-B) convection scheme (Tiedtke 1989; Bechtold et al. 2008) for use at CP resolutions. It was tested for a predominantly shallow convective day in a domain that covers Germany and surrounding regions (Sakradzija and Klocke 2018) to demonstrate that a correct representation of shallow convection can be achieved across the resolution span of $1-10 \mathrm{~km}$. This was demonstrated by the vertical structure of the boundary layer that remained almost unchanged across resolutions and very similar to the reference LES case. In the present study, we show how this stochastic representation of shallow convection interacts with resolved deep convection, clouds and precipitation.

A set of simulations has been performed for 20 December 2013 over the tropical Atlantic and adjacent landmasses, covering the domain from $10^{\circ} \mathrm{S}$ to $20^{\circ} \mathrm{N}$ and from $70^{\circ} \mathrm{W}$ to $15^{\circ} \mathrm{E}$ (see also Fig. 1), which encompasses the shallow convection dominated trade wind regions and the ITCZ region dictated by deep convection. This day was part of the NARVAL-I field study (Stevens et al. 2019), was already simulated in a similar configuration by Klocke et al. (2017) and evaluated in terms of its representation of deep convection by Senf et al. (2018). The relatively short (1-day) duration of the simulations in this study excludes the possibility to examine the remote effect of subgrid shallow convection on the large-scale circulation. On this time scale, no robust effects of the shallow convection in the trades is expected on the upwelling branch of the Hadley cell. The different numerical experiments have been evaluated against satellite observations to assess the performance of different model setups with regard to precipitation and cloud characteristics.

The methods used in this study are described in section 2, including the description of the ICON model, its configurations, the case study used for the process level investigation (section 2a) and the description of the observations (section $2 \mathrm{~b}$ ). The results of the numerical experiments are presented in section 3. The explanation of the results and possible causes of the observed impact of the local shallow convection on clouds and precipitation are discussed in section 4. Section 5 summarizes and draws conclusions of the study. 


\section{Methods}

\section{a. Description of ICON and its configurations}

The version of the ICOsahedral Nonhydrostatic (ICON) model used in this study is the operational code version ${ }^{1}$ as of January 2019 of the Deutscher Wetterdienst (DWD), the national weather service of Germany. The model solves the nonhydrostatic equations on an icosahedral-triangular Arakawa $\mathrm{C}$ grid. The prognostic variables include the horizontal velocity component normal to the triangle edges $v_{n}$, the vertical wind component $w$, density $\rho$, virtual potential temperature $\theta_{v}$, water vapor, and five hydrometeor categories. The time integration is solved using a predictorcorrector scheme. The spatial differences are approximated using a second order method, while horizontal advection is solved by a fifth order method. The continuity equation is discretized using a finite-volume scheme, which inherently guarantees mass conservation. The horizontal differences are solved explicitly, while the vertical differences are treated implicitly by a horizontally explicit-vertically implicit time-stepping scheme. For more details about ICON see Zängl et al. (2015).

The parameterizations of subgrid processes are inherited from the COSMO model (Baldauf et al. 2011) and from the integrated Forecasting System (IFS) of the European Centre for Medium-Range Weather Forecasts (ECMWF). In particular, turbulence is parameterized by the scheme of Raschendorfer (2001) based on a prognostic equation for turbulent kinetic energy (TKE), the land surface by the TERRA scheme (Heise et al. 2006), cloud microphysics by the COSMO-DE scheme (Reinhardt and Seifert 2006; Seifert 2008), a diagnostic cloud cover scheme and the radiative transfer as in Mlawer et al. (1997). The applied cloud microphysics treats two liquid categories (cloud droplets, rain) and three cloud ice categories (ice, snow, and graupel) within a one-moment scheme. The convection scheme based on Tiedtke (1989) as described in Bechtold et al. (2008), here the Tiedtke-Bechtold (T-B) scheme, is used for shallow convection. In fact, the T-B scheme is designed to treat all types of convection in a single framework and thus is activated initially for all convectively unstable grid cells (see sections $3 \mathrm{~b}$ and $3 \mathrm{e}$ ). However, tendencies calculated for midlevel and deep convective grid cells (as defined by the $\mathrm{T}-\mathrm{B}$ scheme) are discarded and these convective scales are left to be explicitly resolved by the model dynamics. The T-B scheme is further developed to include the stochastic shallow convection of Sakradzija et al. $(2015,2016)$ with the parameters and related settings as in Sakradzija and Klocke (2018). The part of the convection scheme that calculates subgrid rain is deactivated in the stochastic version of $\mathrm{T}-\mathrm{B}$ due to inconsistencies between the stochastic sampling and the microphysical relations that were initially defined for the bulk convective scheme.

\footnotetext{
${ }^{1}$ branch icon-nwp/icon-nwp-dev-stochconv based on icon-nwp/ icon-2.3.0-nwp4
}

\section{1) Stochastic SHALlOW CONVECTION}

The stochastic scheme used in this study represents a canonical shallow-cloud ensemble based on the theory of Craig and Cohen (2006). It includes stochastic cloud sampling that is constrained by larger-scale physical processes and keeps the memory of subgrid cloud life cycles. At the CP resolutions, a model grid column can only contain a limited number of clouds specified by the total mass flux that can fluctuate about the average mass flux of the cloud ensemble, which is constrained by the imposed mesoscale atmospheric conditions. The aim of the stochastic scheme is to represent such variability of the convective mass flux due to insufficient sampling at the CP resolutions and thereby correct the mass-flux distribution produced by the underlying $\mathrm{T}-\mathrm{B}$ scheme. The mass flux distribution is discussed in more detail in appendix A. The scheme samples shallow convective clouds using two random processes:

1) A process of sampling random variates from a Poisson distribution to set the number of new clouds in a model column $n$ :

$$
p(n)=\frac{G^{n} e^{-G}}{n},
$$

where $G$ is the generating rate of clouds per model time step and grid cell area (see also Craig and Cohen 2006), and

2) A process of sampling random variates from a Weibull distribution to set the lifetime average mass flux $m$ of each of the newly generated $n$ clouds:

$$
p(m)=\frac{k}{\lambda}\left(\frac{m}{\lambda}\right)^{k-1} e^{-(m / \lambda)^{k}},
$$

where $k$ and $\lambda$ are the shape and scale parameters of the Weibull distribution. Two modes of this distribution are defined for the shallow clouds, one to represent passive shallow clouds and second to represent the active and forced cloud group following the cloud classification of Stull (1985). We leave out the bimodal formulation here for simplicity of the description. For the exact formulation, we refer the reader to Sakradzija et al. (2015) and Sakradzija and Klocke (2018).

The average mass flux of a cloud $m$ also determines its lifetime through a positive correlation function as defined in Sakradzija et al. (2015). The life cycle of each cloud is reconstructed to follow a simple concave function along which the instantaneous mass flux $m^{\prime}$ changes at each time step until the end of the cloud life cycle is reached. In this way at each time step, a grid column of the model contains a number of clouds $N$ at different stages of their life cycles specified by $m^{\prime}$. This introduces memory into the parameterization of the subgrid convection. The clouds in each grid column are then represented by a random sum (sum of a random number $N$ of random variables $m_{i}^{\prime}$ ) of the mass flux $M=\sum_{i=1, N} m_{i}^{\prime}$, which varies in time and space to form a distribution of the total mass flux $p(M)$. This distribution is inherently scale-adaptive, as its shape changes with the model resolution through changes in the average total number of clouds contained in a model column. 
For the closure of the set of two equations, Eqs. (1) and (2), the two unknown parameters $G$ and $\lambda$ need to be related to the ensemble average mass flux $\langle M\rangle$ and the average mass flux per cloud $\langle m\rangle$ of the forced and active cloud group. All the other parameters are set to constant values based on the LES studies of Sakradzija et al. (2015) and Sakradzija and Hohenegger (2017) as used in Sakradzija and Klocke (2018). No tuning of the parameters in the stochastic scheme was done for this study.

The ensemble average value of $M$ is constrained by the T-B mass flux closure by assuming that the bulk mass flux $M_{b}$ is equal to the ensemble average mass flux $\langle M\rangle$. The closure of the $\mathrm{T}-\mathrm{B}$ scheme is then applied to spatially averaged input fields, by taking the area covered by the neighboring cells that share one triangle vortex with the current grid cell (13 cells in total). In this way, the T-B closure is applied at a coarser scale for which it was initially defined, and for which the underlying assumptions of the closure are more appropriate. We constrain $\langle m\rangle$ by using a scaling of the boundary layer heat fluxes, where the efficiency of convective moist heat cycle plays a key role (Sakradzija and Hohenegger 2017). For this second constraint, the spatial averaging of the input fields is also performed to obtain a meaningful scaling relation. For more details about the implementation of the stochastic routine into the ICON, see Sakradzija et al. (2016) and Sakradzija and Klocke (2018).

A small difference between the current setup of the stochastic scheme to that of the previous study of Sakradzija and Klocke (2018) is a detail in the test parcel ascent. The version implemented here follows the default T-B scheme used in the IFS and operational ICON where the test parcel for shallow clouds must originate in the lowest model layer for consistency with the operational code. Sakradzija and Klocke (2018) use a slightly modified version where the test parcel for shallow clouds may also originate from model layers above the surface.

\section{2) MASS-FLUX LIMITERS}

The Tiedtke-Bechtold scheme inherits a set of mass flux limiters from the original implementation in the IFS that limit the magnitude of the cloud base mass flux. These are necessary in the context of long time steps to maintain numerical stability, prevent excessively large tendencies and a depletion of the subcloud layer through convective transport. In the operational ICON implementation, a flow-dependent limiter is set based on the Courant-Friedrichs-Lewy (CFL) criterion. Dependent on the horizontal resolution, the value of the limiter lies between 1 and 2 times the CFL criterion. In addition, a hard limit is applied to the cloud base mass flux, which must not exceed $1.75 \mathrm{~kg} \mathrm{~m}^{-2} \mathrm{~s}^{-1}$. While limiters are generally meant to safeguard stability by catching the occasional unphysical outlier, the mass flux limiters in the operational ICON implementation play a much more active role, and are essential to keep the T-B scheme functional, as we show in appendix A.

The mass flux-limiters, however, restrict the activity of the subgrid shallow convection to a great extent and as a result, the subgrid convection has little chance to alter the resolved convective dynamics. Such a limitation is not appropriate in the stochastic setting since one of the main purposes of the stochastic scheme is to invigorate the resolved convective flow
(Sakradzija et al. 2016). Moreover, the limiters are generally imposed on the bulk mass flux value, while the stochastic sampling introduces a randomly selected value from the probability distribution of all the possible mass flux values constrained in the mean by the bulk value, and as such is not compatible with the limiters. Thus, we effectively disable these limiters in the stochastic configurations of the model by setting the related parameters to a very high value of $100 \mathrm{~kg} \mathrm{~m}^{-2} \mathrm{~s}^{-1}$. For the case study in this paper, disabling the limiters did not cause any major instabilities or runtime problems, likely because very large mass flux values occur only rarely, and due to the stochasticity, are unlikely to occur coherently over large spatial areas or persistently in time. In addition, the "slow" physics time step applicable to the convection parameterization in ICON is shorter ( $300 \mathrm{~s})$ than in many of the IFS configurations used until recently. Thus, the stochastic approach enables us to deactivate the mass-flux limiters and show full functionality of the shallow convection scheme without any artificial limits to its activity and convective strength. In this way, the stochastic scheme introduces a twofold improvement, directly by correcting the mass flux distribution and indirectly by allowing deactivation of the mass flux limiters.

\section{3) THE MAXIMUM ALLOWED SHALLOW CLOUD DEPTH}

The maximum allowed shallow cloud depth $D_{\max }$ is set in the convection code to $200 \mathrm{hPa}$ by default. Clouds that are estimated to grow beyond this depth are considered "deep," and the scheme becomes passive at these grid points, leaving it to the resolved dynamics to represent the convection. The part of the scheme where this estimation of the cloud depth is done is the ascent of a test parcel, a parcel of unsaturated air that rises adiabatically from the surface following a dry adiabat, until it reaches the lifting condensation level. At that point, due to release of the latent heat of condensation the parcel rises following a moist adiabat. The top of the cloud layer is determined as the point where vertical velocity of the parcel becomes less or equal to $0 \mathrm{~m} \mathrm{~s}^{-1}$. We adopt this default value of $200 \mathrm{hPa}$ for $D_{\max }$ in the configurations of the stochastic experiments. However, in the operational T-B configuration a resolution-dependent value of $D_{\max }$ is used, which corresponds to about $77 \mathrm{hPa}$ at the resolution of $2.5 \mathrm{~km}$. This parameter can be used as a tuning parameter to achieve a slightly different solution of the convective dynamics, but also to achieve a more stable model performance. We discovered in the present study that $D_{\max }$ is the most sensitive tuning parameter of the shallow convection scheme, in terms of the interaction between subgrid and resolved convective scales. We have conducted the sensitivity tests based on this parameter shown in appendix B.

\section{4) DECOUPLING OF THE SUBGRID SHALlOW FROM THE RESOLVED DEEP CONVECTION}

In the current operational model setup, the subgrid shallow convection is active regardless of the resolved convective activity. In this case subgrid shallow convection is active up to a vertical limit set by the mixed-layer depth and $D_{\max }$ at the same place and time where resolved convection is present. Only in the case where resolved convection develops a cloud layer deeper than $D_{\max }$, a threshold set in the convection scheme, the 
TABLE 1. List of the model configurations with a short description.

\begin{tabular}{llccc}
\hline \multicolumn{1}{c}{ Description of the settings } & Abbreviation & $M$ limited & $D_{\max }$ & Decoupled \\
\hline $\begin{array}{l}\text { Main experiments } \\
\text { No subgrid convection }\end{array}$ & NOCONV & - & - & - \\
Operational T-B scheme & D-OPER & Yes & $\sim 77 \mathrm{hPa}$ & No \\
Default stochastic & S-NOMFL & No & $200 \mathrm{hPa}$ & No \\
Additional experiments & & & & \\
Decoupled stochastic & S-DCPL & No & $200 \mathrm{hPa}$ & Yes \\
As in S-NOMFL, but disabled in the ITCZ & S-nosh-ITCZ & No & $200 \mathrm{hPa}$ & No \\
As in S-NOMFL, but disabled in the trades & S-nosh-trades & No & $200 \mathrm{hPa}$ & No \\
\hline
\end{tabular}

effects of the parameterization are deactivated in that grid column. Thus, the effects of the shallow convection scheme are still active at the initial stages of the deep cloud development as long as the cloud layer is shallower than $D_{\text {max }}$.

To assess the impact of the shallow convection scheme on the development of the resolved deep convection, we introduce a configuration of the model where the shallow convection scheme is decoupled from the resolved deep convective motion. To achieve this, a threshold of $0 \mathrm{~Pa} \mathrm{~s}^{-1}$ is set in the pressure vertical velocity $\omega$ at a level of about $650 \mathrm{hPa}$ to decide on the shallow convective activity. If the resolved motion develops upward vertical velocity (negative $\omega)$ at this height level, the subgrid shallow convection is deactivated. Such decoupling is used only in one of the stochastic configurations of T-B in this study, S-DCPL [see section $2 \mathrm{a}(5)]$.

In the operational mode used at coarse resolutions, the T-B scheme separates the convection into three categories, shallow, middle, and deep convection, while for the application at $\mathrm{CP}$ resolutions, only the shallow convection category is parameterized. This brings an inconsistency in the treatment of some of the rare points that are initially recognized as deep-convective points, but are in the later stages of the parameterization characterized as shallow convective points and are treated by the shallow convective part of the scheme. At these points, the development of resolved deep clouds is at an early stage where the cloud layer depth still did not reach the threshold of $200 \mathrm{hPa}$ that defines the transition to middle or deep clouds. An adequate massflux closure for these points does not exist. An initial value of the mass flux is instead set to a fraction of the imposed mass flux limit. We find that how these points are treated has an impact on the resolved deep clouds and thus we point to a need to develop a new closure for these early stages of deep cloud development. In the present study, the mass flux value at these points is set to $0.3 \times \Delta p M_{\mathrm{lim}, \mathrm{CFL}} /\left(g t_{\text {conv }}\right)$, while in the T-B operational setup it is set to $0.1 \times \Delta p M_{\text {lim,CFL }} /\left(g t_{\text {conv }}\right)$. Here, $M_{\text {lim,CFL }}$ is the imposed mass flux limit, $\Delta p$ is the depth of the model layer at cloud base in pressure units, $g$ is the gravitational acceleration, and $t_{\text {conv }}$ is the convective time step. This change is introduced to enhance the resolved deep convection, while the low-level clouds and the resolved larger-scale circulations are not affected in any significant way. We are, however, aware that such treatment is rather arbitrary.

\section{5) SETUP OF THE NUMERICAL EXPERIMENTS}

We run several experiments using ICON in a configuration that excludes the parameterization of the midlevel and deep convection, as defined in the T-B scheme. The experiment setup differs by the choice of subgrid shallow convection, activation of limiters of the maximum allowed mass-flux [see section $2 \mathrm{a}(2)$ ], the maximum allowed shallow cloud depth [section $2 \mathrm{a}(3)$ ] and the condition of local coupling or decoupling of the subgrid to the resolved convection [section 2a(4)]. Different model configurations used in this study are summarized in Table 1 and include the following:

1) no shallow convection (NOCONV)

2) the default $T-B$ shallow convection that includes the default operational tuning and active mass flux limiters (D-OPER);

3) stochastic version of the T-B shallow convection, where the mass flux limiters are inactive, including

1) a version with the maximum shallow cloud depth $D_{\max }$ set to $200 \mathrm{hPa}$, and with all tuning parameters in the convective scheme set to their default values (S-NOMFL);

2) a version where shallow convection is deactivated if deep resolved convection is present at that location and time step, i.e., where shallow convection is locally decoupled from deep convection (S-DCPL);

$3)$ and a version where shallow convection is inactive in the ITCZ between $0^{\circ}$ and $10^{\circ} \mathrm{N}$ (S-nosh_ITCZ) or in the trades from $10^{\circ}$ to $20^{\circ} \mathrm{N}$ and from $10^{\circ} \mathrm{S}$ to $0^{\circ}$ (S-nosh_trades);

All simulations are performed using the same horizontal resolution of about $2.5 \mathrm{~km}, 75$ levels in the vertical with a model top at $30 \mathrm{~km}$ and a time step of $20 \mathrm{~s}$. All simulations are initialized at 0000 UTC 20 December 2013 from ECMWF analysis of the atmospheric state and nudged at the lateral boundaries of the simulated domain with the ECMWF 16-kmresolution forecast from that analysis. Simulations are run for $24 \mathrm{~h}$.

\section{b. Observations}

\section{1) Cloud observations}

Cloud observations are obtained from the imaging radiometer Spinning Enhanced Visible and Infrared Imager (SEVIRI) on board the geostationary satellites of the Meteosat Second 
Generation (MSG) series operated by European Organisation for the Exploitation of Meteorological Satellites (EUMETSAT). SEVIRI carries 11 narrow-band channels covering solar and terrestrial radiation with a nadir resolution of $3 \times 3 \mathrm{~km}^{2}$ and one broadband high-resolution visible channel with a 3-times-higher horizontal resolution (Schmetz et al. 2002). For our study we use data from SEVIRI's prime operational service located at a nominal longitude of $0^{\circ}$ and a scan repeat cycle of $15 \mathrm{~min}$. The satellite data has been subsampled to hourly resolution to be comparable to the output frequency of the model.

For model evaluation, Meteosat measurements of visible reflectances at $0.6 \mu \mathrm{m}$ and infrared brightness temperatures (BTs) at various wave lengths are taken. The BTs provide a measure of an effective blackbody emission temperature that can be sensitive to the cloud-top height or atmospheric humidity. In the atmospheric window at $10.8 \mu \mathrm{m}$, atmospheric gases are relatively transparent and the thermal emission comes mainly from Earth's surface, cloud tops, or a combination of both. Low (cold) BTs are found for cirrus clouds and high (warm) BTs occur in cloud-free regions or show the presence of very low clouds. The visible $0.6 \mu \mathrm{m}$ channel provides additional information on the optical thickness of the clouds.

Using the information from the different infrared SEVIRI channels, a cloud classification is obtained from the application of the NWCSAF software, version 2013. It derives a categorical classification for all cloudy pixels using a set of several multispectral tests (Derrien and Le Gléau 2005). The applied tests depend on viewing geometry, illumination, the geographic location and numerical forecast data. For the latter, short-term IFS forecasts are supplied. Cloud types are divided into different categories depending on their cloud-top height and opacity. In contrast to the International Satellite Cloud Climatology Project (ISCCP) approach (see, e.g., Rossow and Schiffer 1999), the method also works at nighttime. However, as opacity information is derived from infrared channels only, it is less accurate. Opaque clouds are divided into five cloud height classes: very low, low, midlevel, high, and very high clouds that are approximately separated by the pressure levels of $800,650,450$, and $300 \mathrm{hPa}$. Four additional classes distinguish between fractional clouds and high cirrus clouds with different opacity levels called: semitransparent thin, semitransparent moderately thick, and semitransparent thick. No further distinction between convective and stratiform cloud structures is made by the classification algorithm. In the following considerations, fractional clouds are combined with very low clouds, because their separation appears to be rather artificial. To this end, we arrive at eight cloud types utilized for further analysis.

\section{2) SyNTHETIC OBSERVATIONS}

The simulated ICON data has been transformed into the observational space using forward operators. For our study, we apply the forward operator VISOP to derive visible SEVIRI reflectances and the SynSat operator after Keil et al. (2006) and Senf and Deneke (2017) to obtain infrared SEVIRI BTs.

VISOP was developed for the model evaluation study by Heinze et al. (2017) and is based on MFASIS (Scheck et al. 2016), a fast method relying on compressed reflectance lookup tables. The operator takes subgrid clouds and their overlap into account, includes a parallax correction to account for slant viewing angles and an approximation for 3D radiative effects related to inclined cloud tops (Scheck et al. 2018). Effective particle sizes for water and ice clouds are determined using the parameterizations by Martin et al. (1994) and McFarquhar et al. (2003), respectively.

In the SynSat operator, vertical profiles of atmospheric temperature, humidity, condensate content, and subgrid-scale cloud cover are needed as input. Together with several surface variables, the profiles are provided to the RTTOV model (Saunders et al. 1999; Matricardi et al. 2004), here version 11.3, to perform single-column radiative transfer calculations. A standard configuration is applied that has been successfully utilized for ICON simulations in previous studies (Heinze et al. 2017; Senf et al. 2018; Pscheidt et al. 2019). For this, diagnostic subgrid-scale cloud condensate content is added to its gridscale counterpart. Furthermore, ice and snow masses are simply combined to a frozen condensate content and its radiative properties are estimated using relations for randomly oriented hexagonal columns after Fu (1996) and McFarquhar et al. (2003). The derivation of synthetic BTs is impacted by uncertainties in the formulation of microphysical and radiative hydrometeor properties. Considering these issues and typical parameter variations, Senf and Deneke (2017) showed that uncertainties in BTs are a few kelvins and largest for semitransparent cirrus clouds with emissivities close to 0.5 .

The synthetic infrared BTs are also forwarded to the NWCSAF software. A cloud classification is derived from the synthetic satellite data that is directly comparable to the cloud classification obtained from the observations. The fractional coverage of the different cloud types is considered as one of the model evaluation metrics. Further details on the cloud classification approach can be found in Senf et al. (2020) where the method was developed and applied to investigate cloudradiative effects over the North Atlantic.

\section{3) Precipitation}

The precipitation rate in the tropical Atlantic produced by the ICON simulations is validated by comparing it to the 3 hourly real-time multisatellite precipitation analysis of the Tropical Rainfall Measuring Mission (TRMM 2011). The instruments aboard the TRMM satellite include the precipitation Radar operating at $13.8 \mathrm{GHz}$, a nine-channel passive microwave radiometer, and a five-channel visible and infrared radiometer. The product we use is the $3 \mathrm{~B} 42$ created in the "Version 7 TRMM Real-Time Multi-Satellite Precipitation Analysis." The spatial resolution is $0.125^{\circ}$. The precipitation data are collected every three hours with the 3 -h period centered at the synoptic observation hour. The comparison of the modeled to the observed daily averaged precipitation rates is done for the time period of $21 \mathrm{~h}$ starting at 0130 UTC, by collecting the TRMM analysis files from 0300 to 2100 UTC.

\section{Results}

\section{a. Cloud cover and cloud types}

We compare the performance of the ICON model in three main configurations, NOCONV, D-OPER, and S-NOMFL 
(Figs. 1b-d), to satellite observations (SEVIRI, Fig. 1a). The case NOCONV overestimates cloud cover in a large portion of the domain, including the ITCZ region, the shallow convective region in the northwest (NW), and the region covered by lowlevel clouds in the southeast (SE). D-OPER follows by slightly reducing the cloud cover but insufficiently, so it remains very similar to NOCONV. The stochastic case S-NOMFL on the other hand, shows an overall reduction in the cloud cover, especially in the shallow-convective NW region and to the south of the ITCZ (Fig. 1d).

Time series of cloud cover show a delay in the diurnal cycle in NOCONV and D-OPER, while S-NOMFL follows the observed diurnal cycle closer (Fig. 2a). NOCONV largely overestimates the average cloud cover throughout the day by more than $20 \%$, while the other cases sequentially reduce the cloud cover by each improvement added to the model: including the shallow convection in D-OPER and adding the stochastic scheme in S-NOMFL. In the stochastic case, the cloud cover is significantly reduced and more closely matches the observations. The overestimation of cloud cover in NOCONV and D-OPER can also be anticipated from Fig. 2b. The NOCONV and D-OPER experiments possess a BT peak at around $288 \mathrm{~K}$, which corresponds to the cloud-top temperature of shallow marine clouds. The improvement brought by the stochastic scheme is again remarkable, judged by the match in the S-NOMFL and SEVIRI histograms for which the BT peak shifts to values around $295 \mathrm{~K}$ representing the cloud-free ocean.

Figures 3 and 4 show the comparison of categorized cloud cover by different cloud types between different model configurations and the observed cloud type categories. The very low and fractional cloud types are showing the most improvement by the S-NOMFL case, which is closer to observations by approximately $20 \%$ of cloud cover on average than the NOCONV and D-OPER cases (Fig. 4). This was an expected result because these types correspond mostly to the shallow convective clouds in the NW region and scattered shallow convective clouds in the remaining part of the domain for which the stochastic scheme was designed. Second, the low cloud category shows the most improvement in S-NOMFL by reduction of almost $10 \%$ of the cloud cover compared to NOCONV and D-OPER (Fig. 4). Such a significant improvement in low, very low and fractional cloud types is evident in the maps of cloud types (Fig. 3) as a vast reduction in spatial coverage by these two cloud types overall in the domain. Other cloud types do not show significant changes in S-NOMFL compared to NOCONV and D-OPER (Fig. 4).

\section{b. Vertical structure of the tropical troposphere}

The stochastic version of the $\mathrm{T}-\mathrm{B}$ convection scheme efficiently mixes air in the tropical boundary layer (BL) and transports moisture higher up. This makes the BL dryer and slightly warmer and the air above the BL moister in the stochastic case (S-NOMFL) compared to the other cases (NOCONV and D-OPER, Fig. 5a). As a result, the surface turbulent latent heat flux is significantly increased in S-NOMFL (Fig. 6). Stronger heating of the BL by the stochastic convection scheme (Fig. 8e) is countered by a reduction in the sensible heating from the surface (Fig. 6), such that the BL does not get a)

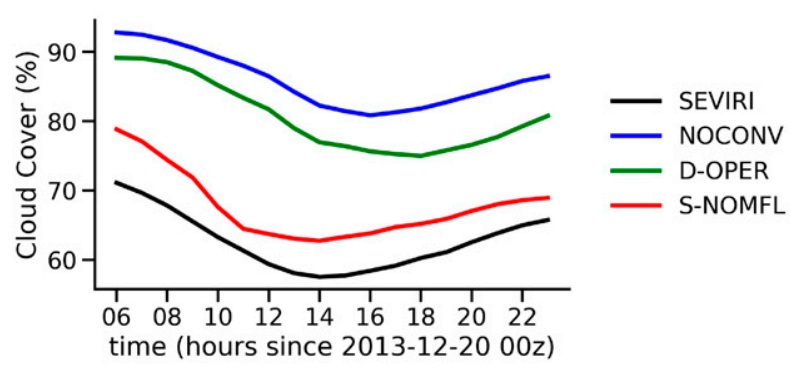

b)

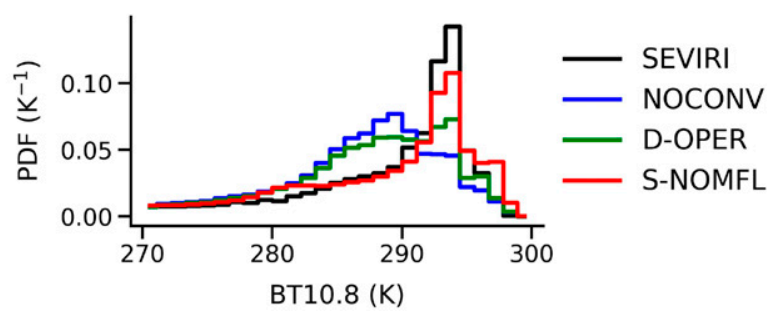

FIG. 2. (a) Time series of the domain-average cloud cover in the tropical Atlantic derived from the NWCSAF cloud mask. The cloud masking has been applied to observational BTs (black line) and the synthetic BTs from the different numerical experiments (colored lines). Cloud-filled and partially cloudy satellite pixels have been counted. (b) Time- and domain-average probability density functions (PDFs) of observed and synthetic BTs at $10.8 \mu \mathrm{m}$ are shown. The PDFs have been normalized in the range between 200 and $300 \mathrm{~K}$, but only a subrange is shown.

much warmer in S-NOMFL compared to the other cases, especially not near the surface. These effects of the stochastic scheme on the vertical thermodynamic structure are similar in the three selected regions: the ITCZ region covering from $20^{\circ}$ to $30^{\circ} \mathrm{W}$, its southern flank from $2^{\circ}$ to $4^{\circ} \mathrm{N}$ and its northern flank from $6^{\circ}$ to $8^{\circ} \mathrm{N}$; the NW trade wind region covering from $8^{\circ}$ to $20^{\circ} \mathrm{N}$ and from $40^{\circ}$ to $65^{\circ} \mathrm{W}$; and the SE region covering from $10^{\circ} \mathrm{S}$ to $2^{\circ} \mathrm{N}$ and from $20^{\circ} \mathrm{W}$ to $10^{\circ} \mathrm{E}$.

Moisture supply to the BL by evaporation from the ocean's surface is further enhanced by an accelerated horizontal mean flow in the trade winds regions (NW and SE) in S-NOMFL (Fig. 5b). The zonal wind is stronger in the NW region at all heights, while in the SE region it is slightly weaker above the $\mathrm{BL}$. The meridional component of the trade winds is stronger by about $2 \mathrm{~m} \mathrm{~s}^{-1}$ in S-NOMFL in the NW region, while in the $\mathrm{SE}$ region the southward flow in the upper levels is more pronounced. In the northern and southern flanks of the ITCZ, zonal wind is stronger, however, the meridional wind component is weaker in S-NOMFL compared to the other cases. In these experiments, changes in the momentum transport caused by the local subgrid convection in the trades accelerate the trade winds rather than any changes in the strength of the large-scale circulation by invigoration of the updrafts in the ITCZ (see section 3e). We leave the investigation of 

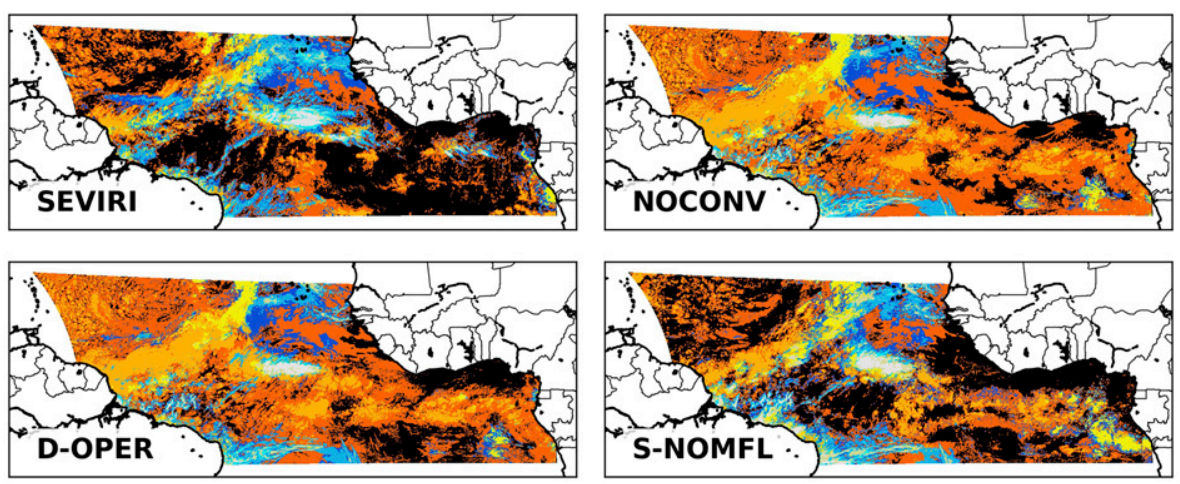

FIG. 3. Overview of the different observed and simulated cloud types derived with the NWCSAF software. The cloud scenery is taken at 1200 UTC 20 Dec 2013.

how the subgrid convection could change the momentum transport of the resolved flow for some future study.

As a result of a more efficient mixing and convective heating of the BL by the stochastic scheme (Figs. 7 and 8), the cloud base is lifted higher up and the cloud fraction is reduced in the trade wind regions in S-NOMFL compared to the other cases (Fig. 5c). In S-NOMFL (Figs. 7c,d), the heating in the boundary layer is much stronger and the boundary layer depth is approximately doubled compared to NOCONV and D-OPER (Figs. 7a,b). When the subgrid shallow convection is not active (NOCONV), heating in the boundary layer is weaker and the boundary layer height is lower compared to S-NOMFL, as shown in the sums of the daily averaged temperature tendencies of all the processes (except bulk microphysics) in Fig. 7. Similar situation occurs when the deterministic subgrid shallow convection is used in the operational configuration. The vertical profiles of all quantities shown in the Fig. 5 do not differ substantially between NOCONV and D-OPER.

To investigate how processes interact among each other and with the resolved flow dynamics, we look into the heating and cooling rates that result from different processes in the simulations. For this analysis, we plot the daily average of the temperature tendencies due to various processes in two longitudinal bands from $40^{\circ}$ to $45^{\circ} \mathrm{W}$ to capture the trade wind region in the $\mathrm{NW}$ of the domain, and from $20^{\circ}$ to $30^{\circ} \mathrm{W}$ where we find the deepest clouds in the ITCZ (Figs. 7 and 8). The strongest forcing results from the shallow convection scheme manifested as a strong heating within the boundary layer including the shallow cloud layer, but also in deeper clouds up to the height of about $5 \mathrm{~km}$ in the NW trade wind region and ITCZ (Figs. 7c,d and 8e). These are congestus clouds that are abundant in the tropics and significantly contribute to the tropical rainfall, moisten and precondition the atmosphere for deep convection (Johnson et al. 1999). The subgrid convective heating in the S-NOMFL case coincides with stronger cooling near the surface due to the resolved dynamics and a wider signature of deep convective cooling in the ITCZ (Fig. 8b) compared to the NOCONV case (Fig. 8a). The NOCONV case exhibits a strong polarity in cooling and warming due to turbulence at the boundary layer top to the south and north from the ITCZ, which is less pronounced in the
S-NOMFL case. The boundary layer is deeper in S-NOMFL compared to the NOCONV case. Thus, shallow convective heating is substantial and it significantly changes the heating and cooling patterns due to dynamics and turbulence in and just above the BL, but also in the deep cloud layer.

\section{c. Precipitation and deep convection}

The precipitation rate over three hours centered at 1200 UTC is plotted in Fig. 9. The precipitation in the ITCZ region is similarly represented using the two ICON configurations NOCONV and D-OPER (Figs. 9b,c) with similar differences compared to

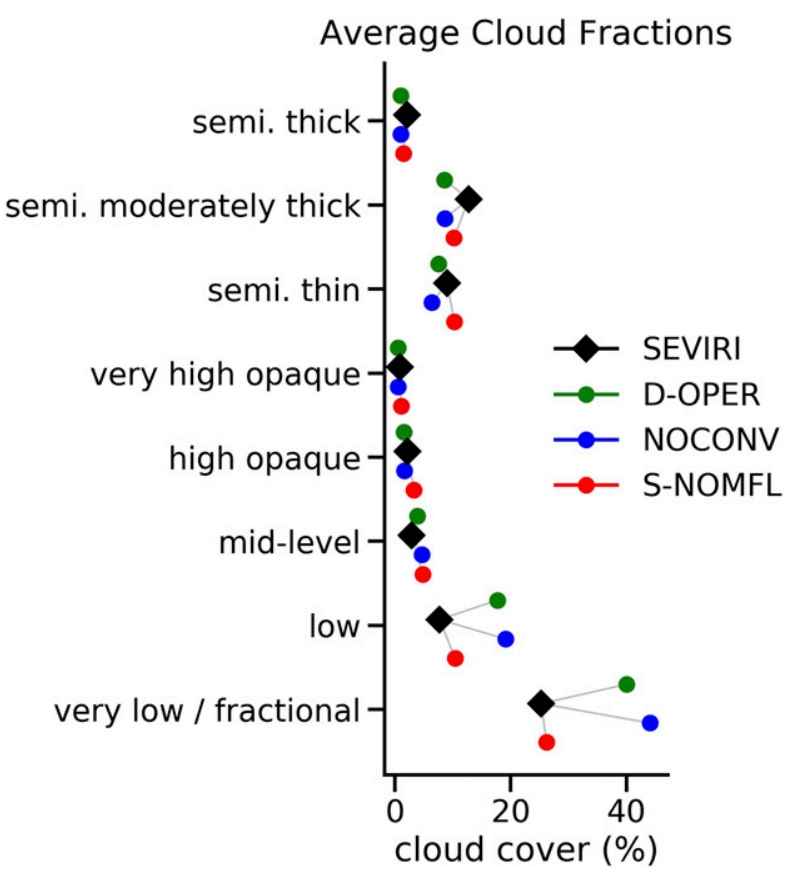

FIG. 4. Domain- and time-average fractional coverage of different cloud types derived with the NWCSAF software. Observed and synthetics BTs have been chosen as a basis for the cloud typing. The results have been vertically stacked per cloud type and observations are presented in black and simulations are presented with colors. 
a) vertical thermodynamic structure
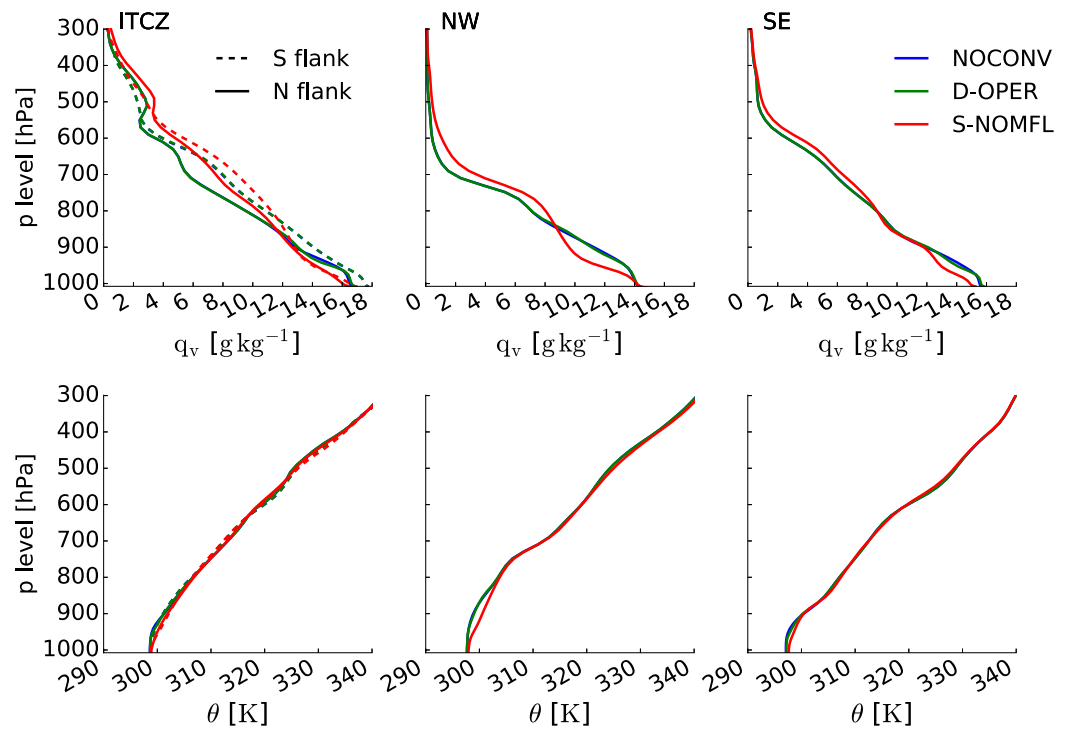

b) horizontal wind components
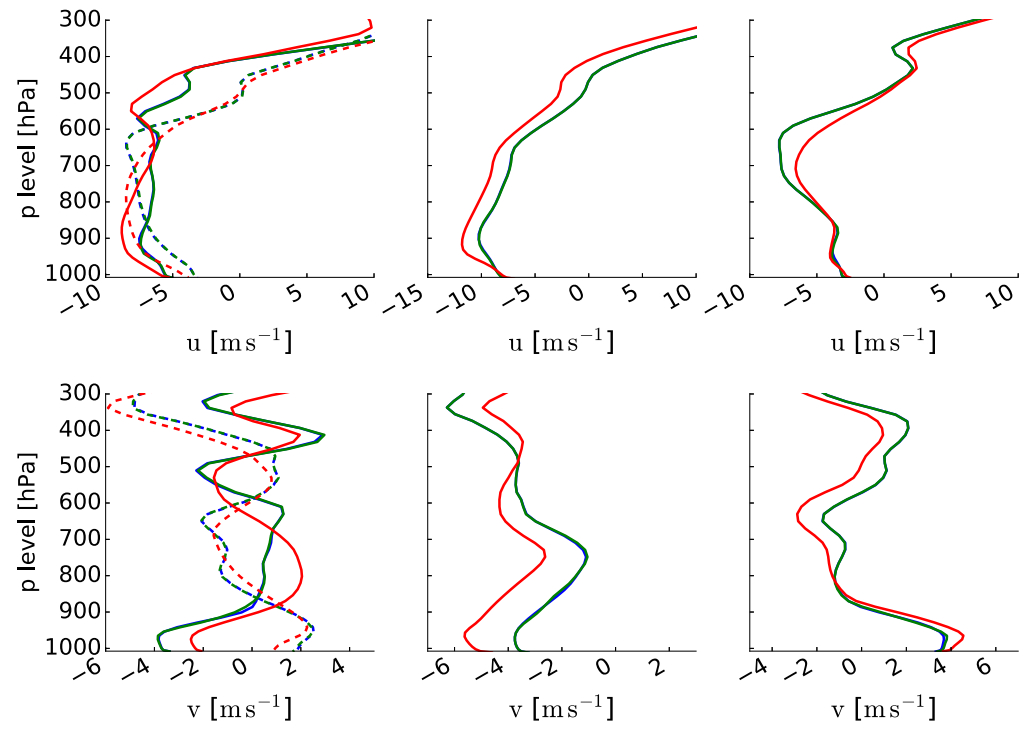

c) structure of the cloud layer
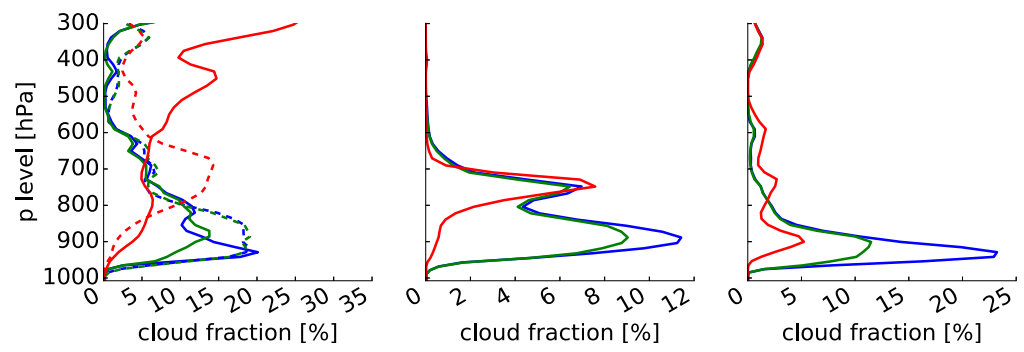

FIG. 5. Vertical profiles of (top to bottom) total water content, potential temperature, wind components, and cloud fraction averaged over the time period from 1200 to 2400 UTC. The ITCZ region covers from $20^{\circ}$ to $30^{\circ} \mathrm{W}$, its southern flank covers from $2^{\circ}$ to $4^{\circ} \mathrm{N}$, and its north flank covers from $6^{\circ}$ to $8^{\circ} \mathrm{N}$; the NW trade winds region covers from $8^{\circ}$ to $20^{\circ} \mathrm{N}$ and from $40^{\circ}$ to $65^{\circ} \mathrm{W}$; and the $\mathrm{SE}$ trade winds region covers from $10^{\circ} \mathrm{S}$ to $2^{\circ} \mathrm{N}$ and from $20^{\circ} \mathrm{W}$ to $10^{\circ} \mathrm{E}$. 

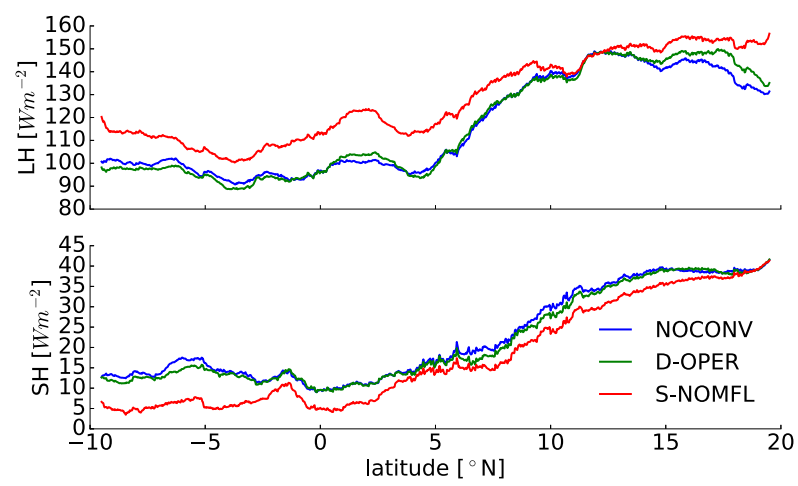

FIG. 6. Zonally averaged surface turbulent heat fluxes, (top) latent and (bottom) sensible heat flux, averaged over the time period from 1200 to 2400 UTC.

the TRMM analysis (Fig. 9a). The case with the most differences compared to observations is D-OPER in which vast regions are covered by a low-rate rain that we do not find in observations ("drizzle bias"). The most striking difference in the precipitation maps is in the NW shallow-convective region, where both NOCONV and D-OPER largely overestimate the rain rates and show the rain patches organized into parallel streaks. Nevertheless, such high rain rates did not reduce the cloud cover in the NW region in these two cases (Figs. 1b,c).

The stochastic version of the T-B scheme was designed to improve the representation of shallow convection and was previously tested on a case situated in the NW trade wind region (Sakradzija et al. 2016). We find that the rain rates in the NW region are slightly reduced in the S-NOMFL case (Figs. 9d,e) compared to NOCONV (Fig. 9b), while keeping the cloud cover at a more realistic level (Fig. 1d). The precipitation rate might still be overestimated in S-NOMFL in this region; however, we refrain from making further conclusions due to a high uncertainty of the TRMM dataset in detecting the low-value precipitation rates (Behrangi et al. 2012). We also find that S-NOMFL overpredicts the rain rates in the $\mathrm{SE}$ region between $2^{\circ} \mathrm{S}$ and $2^{\circ} \mathrm{N}$ where two rainbands are formed. This overprediction of the rain is most probably the result of an overestimation of the bulk mass flux in this region. Since we cannot compare the mass flux to the observations in this region, we made a relative comparison of these results to an experiment where the mass flux is reduced in this region and that does not show such overprediction and doubled rainband (not shown).

Zonally averaged daily precipitation rate for the latitude range $2^{\circ}-8^{\circ} \mathrm{N}$, as a proxy for the ITCZ position and width, is reproduced well only in the S-NOMFL case (Fig. 10). In this case, the width and peak of the precipitation band approximately follow the observed TRMM signature. The width and location of the precipitation band in S-NOMFL coincide with the width and location of the convective heating in the BL and up to the height of about $5 \mathrm{~km}$ between approximately $3^{\circ}$ and $8^{\circ} \mathrm{N}$ (Fig. 7). As in the previous plots, NOCONV and D-OPER perform similarly and produce a shift in the ITCZ location, with a precipitation peak moved to the south by approximately $1^{\circ}$ of latitude, while its width is also reduced compared to
TRMM. In NOCONV and D-OPER, even though the subgrid convective activity is very low, the precipitation band is narrowly centered around the peak BL heating activity around $5^{\circ} \mathrm{N}$. All cases fail to reproduce the precipitation peak between $9^{\circ}-11^{\circ} \mathrm{N}$.

The cold cloud structures seen in Fig. 11 are partially formed by deep convective motion and therefore appear at places where also precipitation is strong. All numerical experiments are able to simulate the observed large cold cloud cluster in the center of the tropical Atlantic, which is connected to a positive, synoptic-scale moisture perturbation. However, as shown in a previous study, the sizes of the large cloud clusters are underestimated by the ICON simulations (Senf et al. 2018), which also leads to an underestimation of the occurrence frequencies of very cold BTs (see Fig. 11 bottom). Observed BT occurrence frequencies decay rather linearly toward very low BT values. Simulated occurrence frequencies decay much faster, especially for the stochastic configuration.

\section{d. Interaction of shallow convection with the resolved circulations}

The stochastic convection invigorates the resolved convectivescale flow in the boundary layer. Peak upward and downward motions at $900 \mathrm{hPa}$ are approximately twice as strong in S-NOMFL compared to NOCONV and D-OPER (Fig. 12a). We look into three regions separately, namely, the ITCZ region covering from $2^{\circ}$ to $8^{\circ} \mathrm{N}$ and from $20^{\circ}$ to $30^{\circ} \mathrm{W}$, and the NW and $\mathrm{SE}$ trade wind regions as defined in section $3 \mathrm{~b}$. In the ITCZ, mainly updrafts are invigorated by the stochastic convection, while the effect on the downdrafts is smaller. In the NW and SE trade wind regions, updrafts are more invigorated than downdrafts, but both are stronger compared to NOCONV and D-OPER.

As indicated by comparing vertical cross sections of the zonally averaged pressure velocity (Fig. 12c), the region covered by updrafts in the ITCZ is substantially broader in width in S-NOMFL as compared to NOCONV and D-OPER. A wide updraft region exists between approximately $3^{\circ}$ and $7^{\circ} \mathrm{N}$ in S-NOMFL, while it is narrower between $4^{\circ}$ and $6^{\circ} \mathrm{N}$ in NOCONV and D-OPER. The wider and more intermittent updraft region results from a more intermittent behavior of the convection scheme when the mass flux limiters are deactivated, which alone produces unrealistic convection and its spatial distribution (see D-NOMFL in appendix A). As counterintuitive as it seems, the stochastic version of the convection scheme reduces such intermittency of the subgrid convection when the mass flux limiters are not active. This reduction occurs first by reducing the mass flux values by the stochastic sampling that prevents an "on-off" behavior of the scheme (see appendix A), second by including convective memory at the subgrid scale and third by using the input averaged over the neighboring grid cells [see section $2 \mathrm{a}(1)$ ]. A broader precipitation band in S-NOMFL compared to NOCONV and D-OPER (Fig. 10) is closely related to the wider updraft region in S-NOMFL.

\section{e. Isolating local interactions}

Are the differences in simulated precipitation and deep convection in the ITCZ, as discussed in section $3 \mathrm{c}$, due to remote or local effects of subgrid shallow convection? To answer 
a) NOCONV western band

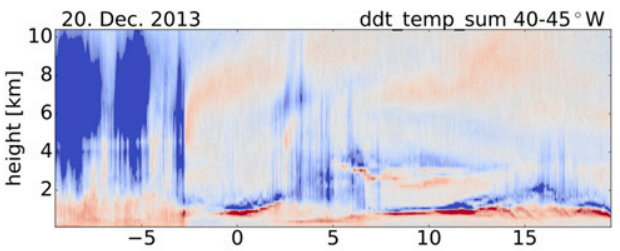

c) S-NOMFL western band

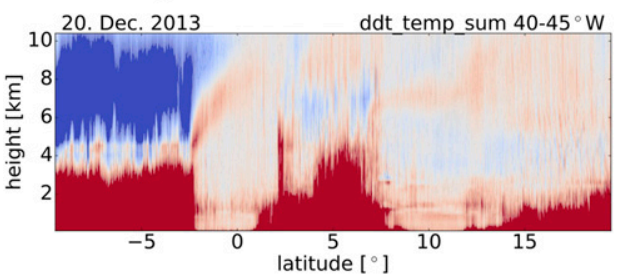

b) NOCONV central band

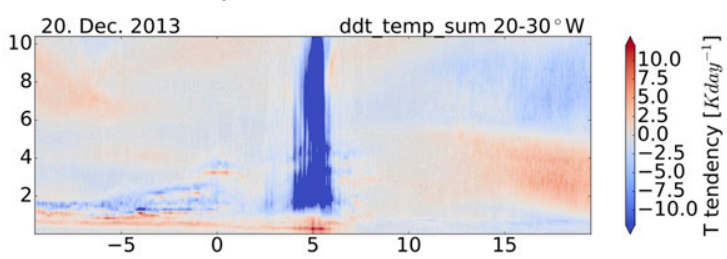

d) S-NOMFL central band

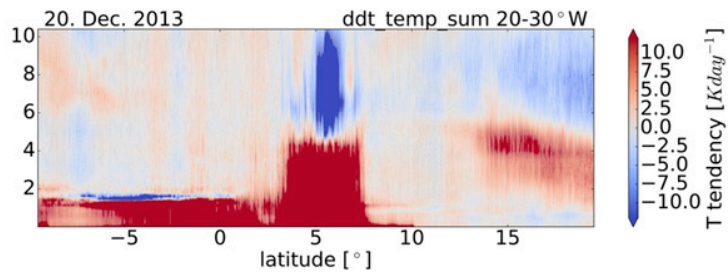

FIG. 7. Daily mean of the sum of temperature tendencies from resolved model dynamics, turbulence, convection, and radiation zonally averaged over two bands, (a),(c) the western band covering $40^{\circ}-45^{\circ} \mathrm{W}$ to demonstrate the activity of the shallow convection scheme in the NW region and (b),(d) the central band covering $20^{\circ}-30^{\circ} \mathrm{W}$ to better demonstrate the activity of the convection scheme in the ITCZ.

this question, we conducted an additional experiment where we deactivated the stochastic convection scheme at the locations where the resolved flow develops deep convective motion [S-DCPL, see section 2a(4) and Table 1]. Local decoupling between shallow convection and deep updrafts (S-DCPL) mostly affects the ITCZ region where we find a bulk of the deep convective clouds. Such decoupling significantly reduces the strength of the updrafts and downdrafts at low levels in the
ITCZ (Fig. 13a, left) and exhibits shallower and more intermittent updrafts compared to all other cases, while the updraft region is kept wide for the same reasons as in S-NOMFL (Fig. 12b). Such weaker and shallower updrafts produce shallower clouds in the ITCZ, which explains the low precipitation rates and a mismatch in the magnitude of the precipitation peak in the ITCZ compared to TRMM (Fig. 13c). This indicates the importance of the interaction between subgrid a) dynamics (NOCONV)

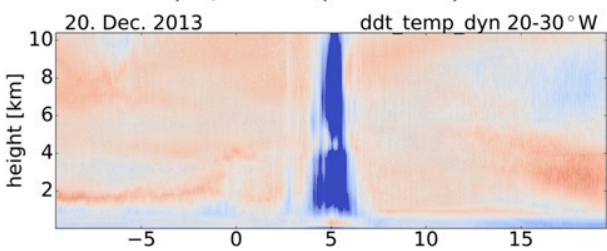

c) turbulence (NOCONV)
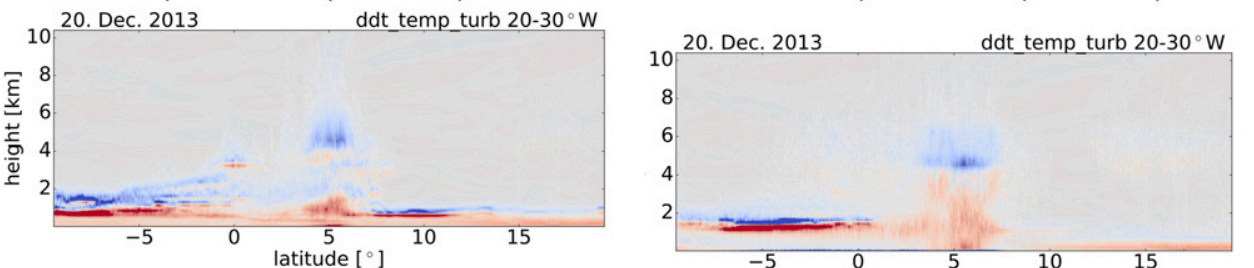

e) convection (S-NOMFL)

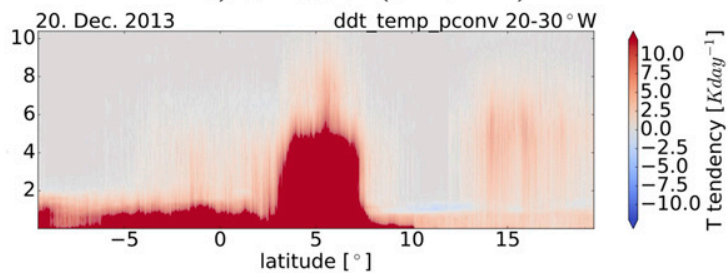

FIG. 8. Contribution of different processes to the daily mean tendencies of temperature averaged over the band $20^{\circ}-30^{\circ} \mathrm{W}$. 
a) TRMM

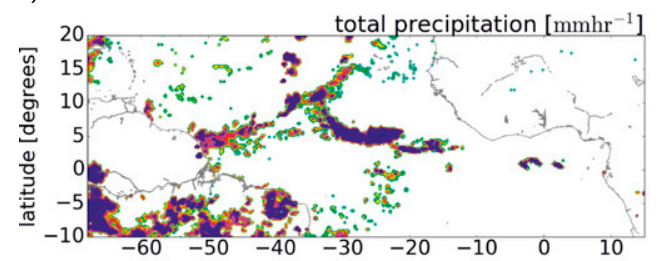

c) D-OPER

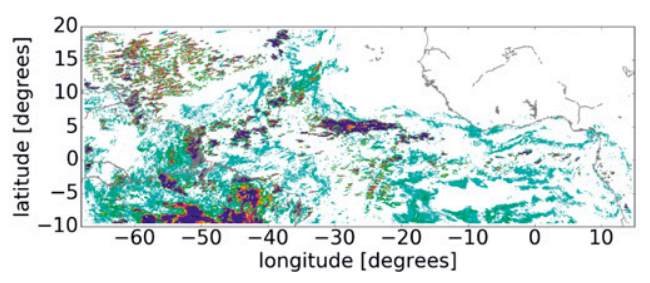

b) NOCONV

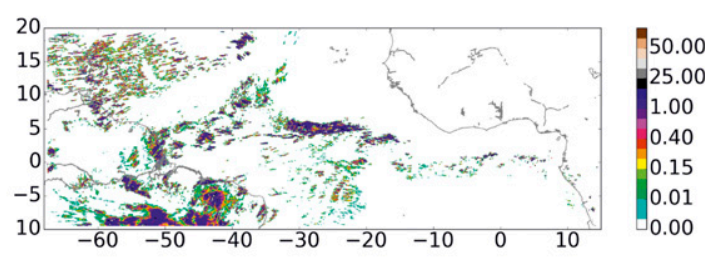

d) S-NOMFL

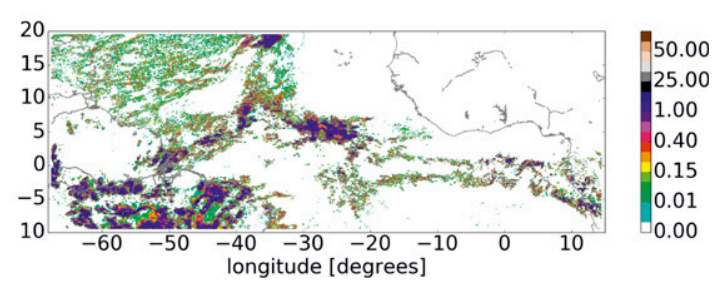

FIG. 9. The 3-hourly precipitation rates centered at 1200 UTC as in the (a) TRMM observations, and ICON model simulation (b) without subgrid convection, (c) using the default operational shallow convection, and (d) using the stochastic version of shallow convection.

shallow convection with the resolved deep convection at the same location and time step in order to reproduce the ITCZ correctly (as in S-NOMFL). Such a local interaction between the subgrid shallow convection scheme and resolved deep convection is a missing ingredient necessary to improve the deep convection and distribution of precipitation in all cases except for S-NOMFL where this interaction is represented at least during the initial stages of deep cloud development.

We also conducted two additional test simulations where we deactivated shallow convection in the ITCZ region (Snosh_ITCZ) or in the trade wind region (S-nosh_trades) to examine the local effects in isolation from any remote effects of shallow convection. As expected, based on the results from the previous experiment (S-DCPL), absence of parameterized shallow convection in the ITCZ (S-nosh_ITCZ) weakens the convective updrafts and downdrafts locally in the ITCZ compared to S-NOMFL (Fig. 13a). Northward wind speed in the ITCZ region is also weaker, as evidenced by the left panel of Fig. 13b. These weaker winds are mostly found to the south of the ITCZ precipitation band (results not shown). In contrast, the lack of parameterized shallow convection in the ITCZ region does not significantly affect the updraft speed and the $v$-wind component in the trades. This result confirms that there is no remote interaction of the invigorated updrafts in the ITCZ region with the trade wind regions through the large-scale circulation on the considered time scales.

Absence of parameterized shallow convection in the trades (S-nosh_trades) weakens the updrafts and downdrafts as well as the horizontal flow $v$ in the NW region and less so in the SE trade wind region (Figs. 13a,b). On the other hand, there is no effect of the absence of shallow convection in the trades on the updrafts in the ITCZ region, and no significant effect on the $v$ wind within the ITCZ. Thus, there is no effective remote interaction between shallow convection in the trades and convection in the ITCZ in these experiments. These results indicate that the lack of parameterized shallow convection in NOCONV is the reason for weaker updrafts (Fig. 13a) and weaker horizontal winds in the trades compared to S-NOMFL (Fig. 5b).

Zonally averaged daily precipitation rate is not well reproduced in the S-nosh_ITCZ case where the precipitation band is too narrow and displaced to the south (Fig. 13c). The signature of the precipitation rate in the ITCZ is similar to the NOCONV case (Fig. 10). This links the misrepresentation of the precipitation band in the NOCONV case to the missing effects of the parameterized shallow convection within the ITCZ. In the $\mathrm{S}$-nosh-trades case, the precipitation band is broader and located more toward north, which matches the TRMM analysis better (Fig. 13c). The latter signature of precipitation in the ITCZ is similar to the signature in S-NOMFL. This links the improved representation of the precipitation band in the ITCZ to the better shallow convection within the ITCZ, regardless of the missing shallow convective activity in the trades.

\section{f. How robust is the improvement due to a local impact of stochastic shallow convection?}

Ten additional days, starting from 10 to 19 December 2013, are simulated using the ICON model in two configurations, NOCONV and S-NOMFL, to assess the robustness of our

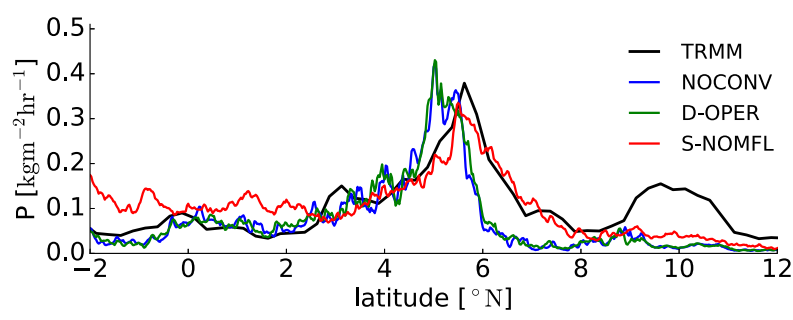

FIG. 10. Zonally averaged precipitation rate accumulated over the day in the four ICON model simulations compared to the TRMM observations. 

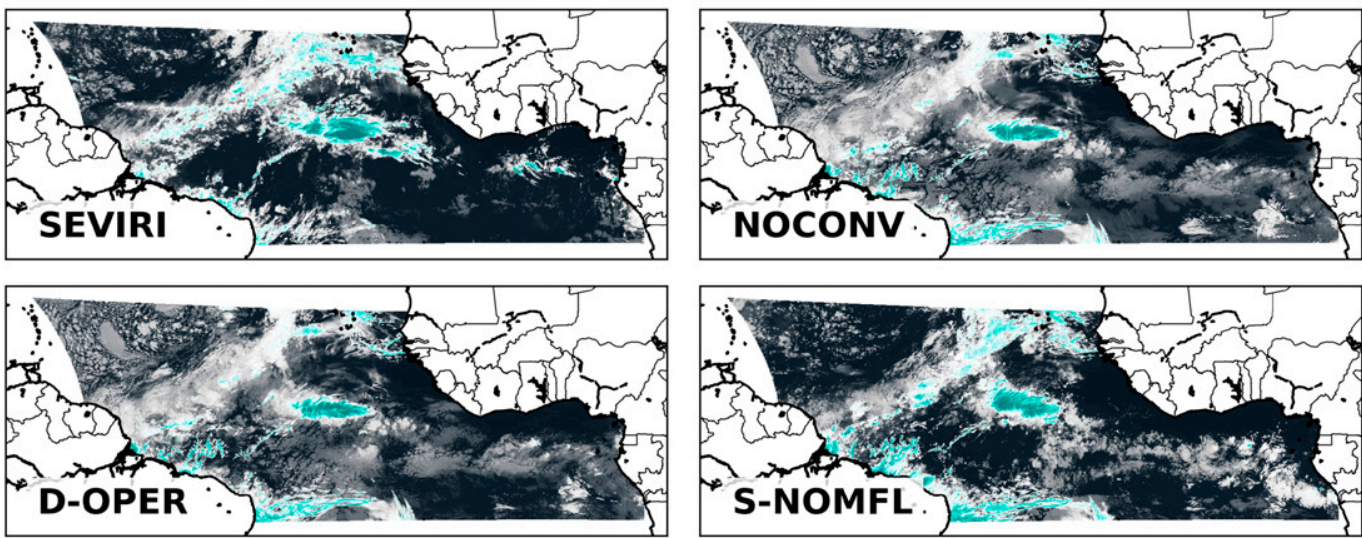

220

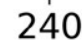

\section{0}

BT10.8 (K)

280

300

b)

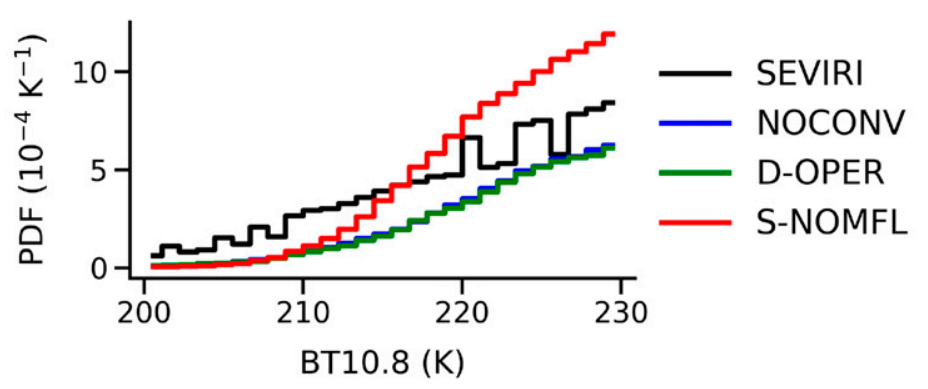

FIG. 11. (a) Spatial distributions of observed and synthetic BTs at $10.8 \mu \mathrm{m}$ at 1200 UTC. The color scale was chosen to highlight cold cloud structures partially resulting from deep convective outflow. The cloud scenery is similar to Fig. 3. (b) Time- and domain-average PDFs of observed and synthetic BTs at $10.8 \mu \mathrm{m}$ are shown. The PDFs have been normalized in the range between 200 and $300 \mathrm{~K}$, but only a subrange is shown.

findings. The simulations are a set of consecutive 1-day simulations to isolate the local effects of parameterized shallow convection from its remote effect through a possible invigoration of the large-scale circulation. The model configurations do not differ from the base case, although we run two sets of experiments in the S-NOMFL setup to examine the effect of a factor of 0.1 versus 0.3 in the mass flux closure for the rare points initially recognized as deep convective [as discussed in section $2 \mathrm{a}(4)]$.

As a measure of robustness, we examine the differences in the total cloud cover and the zonally averaged daily precipitation rates (Fig. 14). Similar to the one-day case study, the stochastic scheme reduces the total cloud cover by $10 \%$ to $20 \%$ compared to the NOCONV case. The improvement brought by the stochastic scheme is thus robust. We also find that the change in the precipitation band is systematic-S-NOMFL produces a wider precipitation band and in most days it is closer to observations compared to NOCONV. It, however, cannot correct the position of the ITCZ in the days where it is set more to the south compared to TRMM most probably already set by the initial conditions, such as 15 and 17 December 2013 (not shown separately). A short span of the simulation does not allow for the large-scale dynamics to act upon the ITCZ location and it cannot be altered solely through the local impact of shallow convection. S-NOMFL generally produces higher precipitation rates compared to NOCONV, while the ITCZ precipitation band gets wider with a change in the mass-flux closure from $0.1 \times \Delta p M_{\mathrm{lim}, \mathrm{CFL}} /(g t)$ to $0.3 \times$ $\Delta p M_{\mathrm{lim}, \mathrm{CFL}} /(g t)$.

\section{Discussion}

The leading cause of the differences we find between modeled clouds and precipitation in the NOCONV case and observations is the finite model resolution of $2.5 \mathrm{~km}$ that prevents the model from resolving the finest convective scales that are 
a) Regional frequency distributions of $\omega$ at around $900 \mathrm{hPa}$
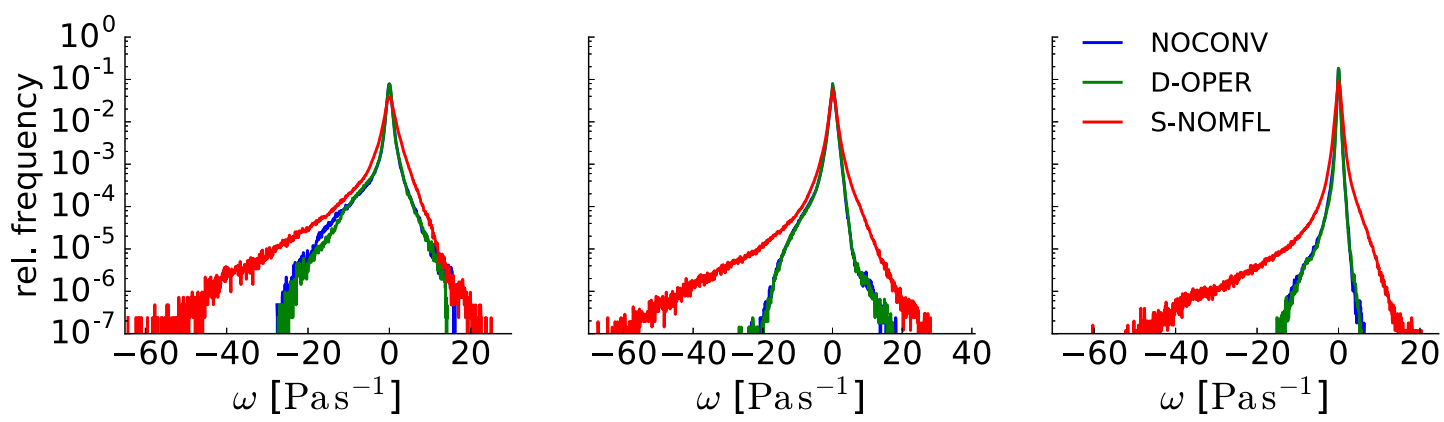

b) Zonally averaged vertical cross-sections of $\omega$

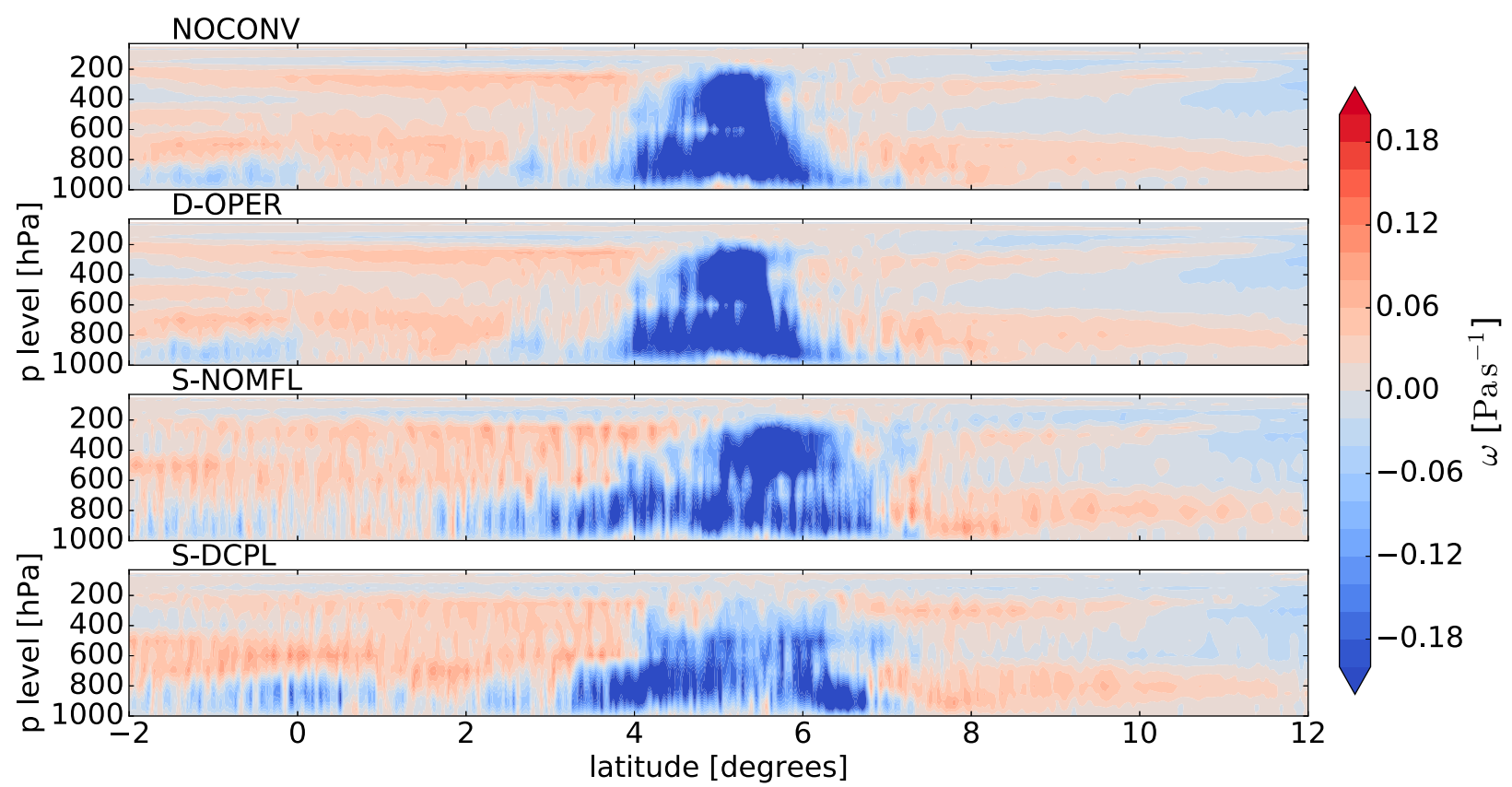

FIG. 12. Frequency of occurrence of (a) the pressure vertical velocity, $\omega$, in the three selected regions: ITCZ region covering from $2^{\circ}$ to $8^{\circ} \mathrm{N}$ and from $20^{\circ}$ to $30^{\circ} \mathrm{W}$, NW region covering from $8^{\circ}$ to $20^{\circ} \mathrm{N}$ and from $40^{\circ}$ to $65^{\circ} \mathrm{W}$, and SE region covering from $10^{\circ} \mathrm{S}$ to $2^{\circ} \mathrm{N}$ and from $20^{\circ} \mathrm{W}$ to $10^{\circ} \mathrm{E}$. (b) Daily and zonally averaged vertical cross sections of $\omega$.

present in nature. The effective model resolution is $6-8$ times the grid spacing (Zängl et al. 2015), which limits the ability to fully resolve scales smaller than approximately $15 \mathrm{~km}$. At these scales, shallow convection is not well represented, while deep convective updrafts are underresolved. The convection that is resolved is spatially too broad, too shallow, too weak, too laminar, and overly organized (e.g., Petch et al. 2002; Bryan et al. 2003; Ching et al. 2014; Sakradzija et al. 2016). The resolved circulations being too broad and too weak, are ineffective in mixing the boundary layer air and in transporting the moisture upward (Fig. 5a), which results in an overestimation of low-level cloudiness (e.g., Hohenegger et al. 2020). The boundary layer is less developed and remains shallower compared to S-NOMFL during the entire simulation, with sharp gradients at the BL top (Fig. 7a). Such underresolved convection produces deep clouds that are too shallow (Fig. 11) and an incorrect distribution of precipitation in the tropical Atlantic (Fig. 10).

The case D-OPER differs very little from the NOCONV case. The two cases exhibit similar resolved flow dynamics, and very similar interaction between the physical processes that result in similar heat budgets (Fig. 8). The reason for this is a weak subgrid convective activity due to the imposed limits in the mass flux, which also limits the convective activity spatially, and due to a too-shallow maximum allowed cloud depth. As such, the subgrid shallow convection in D-OPER is neither strong nor active enough to effectively mix the subcloud-layer and transport the moisture upward, which reflects in a too-high low-level cloud amount and the distribution of precipitation that does not closely follow the observations.

The stochastic shallow convection (S-NOMFL) invigorates the resolved convection by heating the boundary layer and 


\section{a) Regional frequency distributions of $\omega$ at around $900 \mathrm{hPa}$}
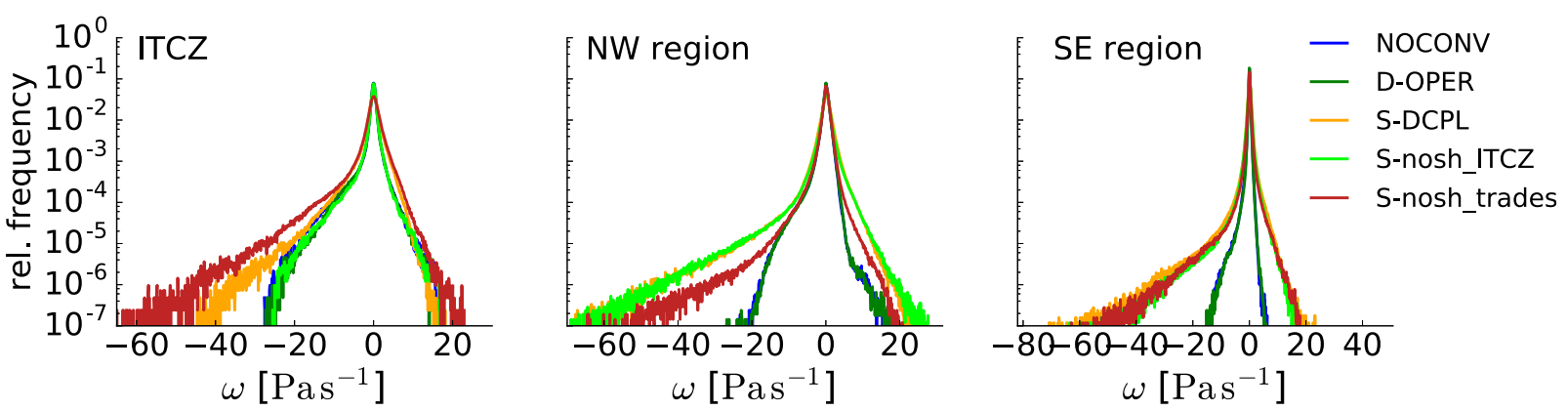

b) Spatial distribution of $v$ at a level of about $450 \mathrm{~m}$ on average
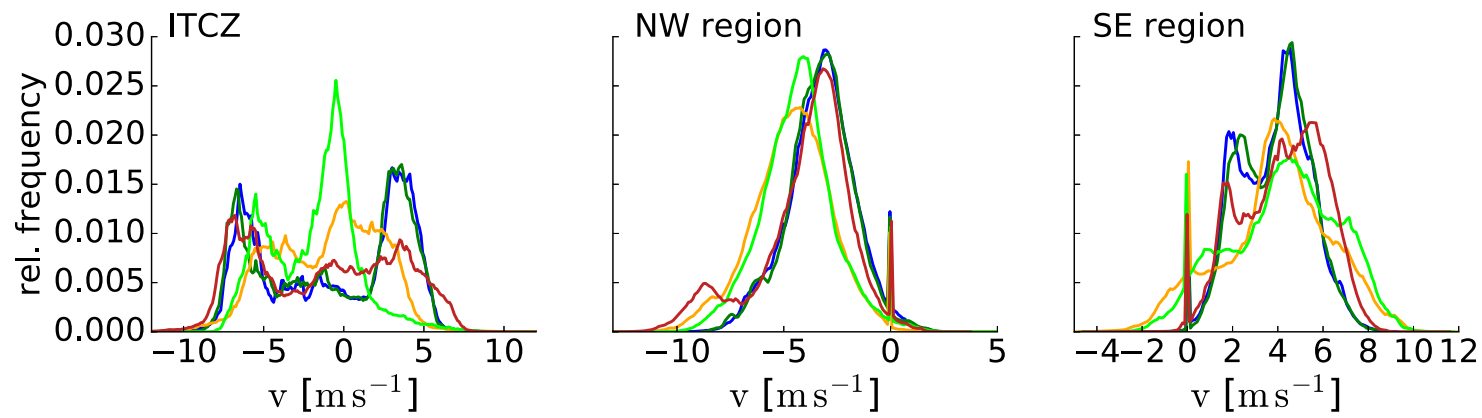

c) Zonally averaged precipitation rate accumulated over the day

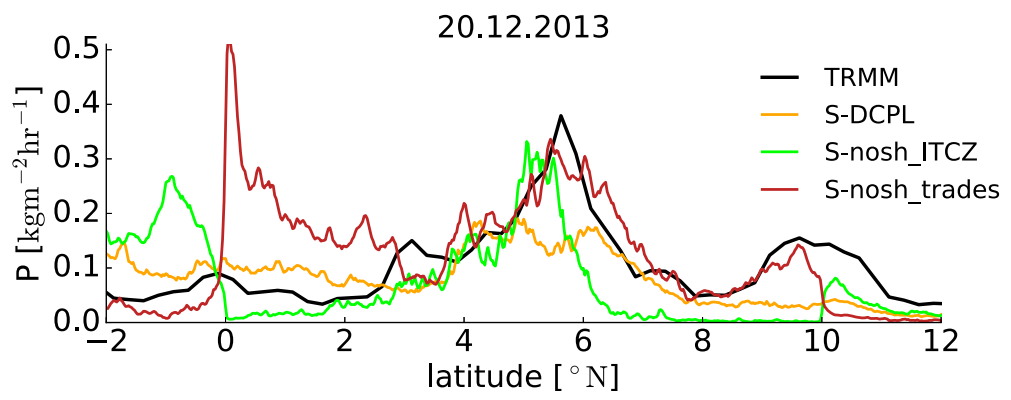

FIG. 13. As in Fig. 9. (a) Frequency distribution of the pressure vertical velocity and (b) meridional wind component at a level of about $900 \mathrm{hPa}$. (c) Zonally averaged precipitation rate in the ITCZ.

increasing the buoyancy of the resolved updrafts. This produces much stronger resolved updrafts and more deep clouds in S-NOMFL compared to D-OPER (Fig. 12b and section 3c). These stronger updrafts efficiently transport moisture upward out of the boundary layer. As a result, the surface sensible heat flux is decreased and the latent heat flux is increased in most parts of the domain (Fig. 6). The subgrid convection has the largest impact on the low-level cloud cover that is reduced by $20 \%$ on average in the stochastic cases.

The subgrid shallow convection scheme also takes the role of a parameterization of deeper congestus type clouds that grow up to the height between 4 and $5 \mathrm{~km}$ even though the parameterized midlevel and deep convection is deactivated. Shallow convective clouds are defined as the clouds that do not develop more than $200 \mathrm{hPa}$ in depth, so the shallow convection scheme is active at all the places where clouds develop until they reach this predefined cloud depth, regardless of their type, shallow, midlevel, or deep. As the cloud base is lifted higher up in the stochastic cases (Fig. 5c), the parameterized cloud top can also reach higher levels, such as the $4-5 \mathrm{~km}$ levels in the ITCZ region and isolated clouds reaching this height in the NW trades.

The bulk mass flux closure of the convection scheme can result in unrealistically high mass flux values at the CP resolutions, which is revealed when the mass-flux limiters are deactivated (D-NOMFL, appendix A). These high mass flux values cause an intermittent behavior of subgrid convection by frequently switching the gridcell state between convectively active and inactive ("on-off" behavior). The stochastic version of the convection scheme applies the closure at a coarser resolution at which the underlying assumptions are still appropriate and resamples the mass flux distribution to adapt to the 
a) total cloud cover

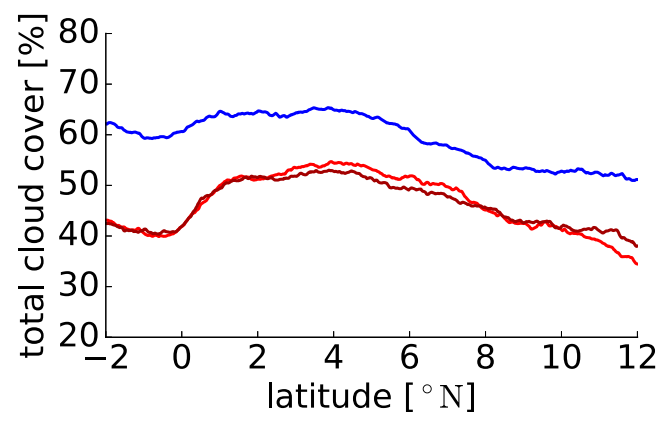

b) total precipitation rate

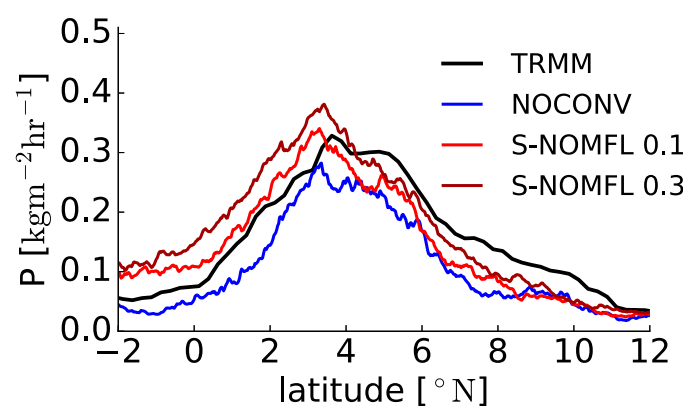

FIG. 14. Zonal average of the (a) total cloud cover (model-based output variable) and (b) daily precipitation rate in the simulated period from 10 to 20 Dec 2013. The case NOCONV is compared to two versions of the S-NOMFL experiment, S-NOMFL 0.1, where the convective mass flux below deep updrafts is estimated as $0.1 \times \Delta p M_{\text {lim,CFL }} /(g t)$ and S-NOMFL 0.3 as $0.3 \times \Delta p M_{\mathrm{lim}, \mathrm{CFL}} /(g t)$ [see section $\left.2 \mathrm{a}(4)\right]$.

CP-resolutions. These two aspects of the stochastic scheme reduce the mass flux values and prevent such on-off behavior. In addition, convective memory introduced through the cloud life cycles results in a more coherent behavior over time compared to the deterministic version when the mass flux limiters are inactive. As a result of the corrected mass flux distribution and convective memory, the stochastic version of the convection scheme invigorates the resolved convection to such an extent to produce realistic clouds and precipitation (Figs. A1-A4).

\section{a) NOCONV}

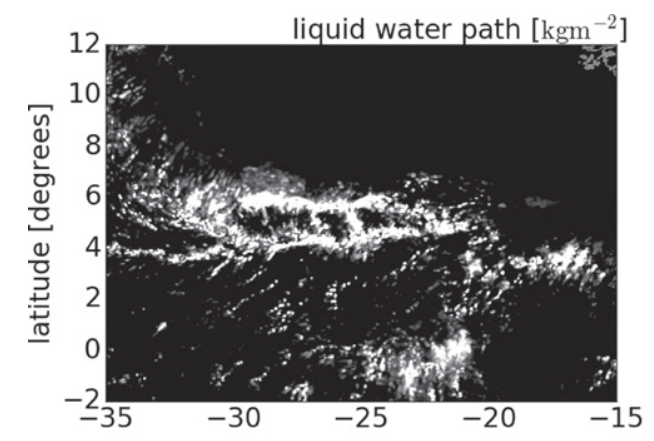

c) S-NOMFL

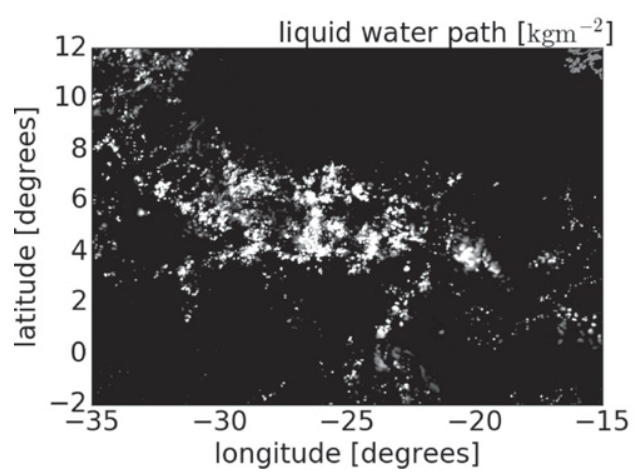

Based on our tests, parameterized local convective heating in the BL below the resolved deep updrafts is responsible for the improved representation of the precipitation band in the ITCZ. Such heating fuels and invigorates the resolved updrafts at the initial stages of the deep cloud development and produces more deep clouds that bring more precipitation. The position and width of the precipitation band of the ITCZ seem to be affected by a qualitatively different spatial organization of deep convective clouds in the four cases. In the NOCONV case, the ITCZ band is composed of two separated parallel

\section{b) D-OPER}

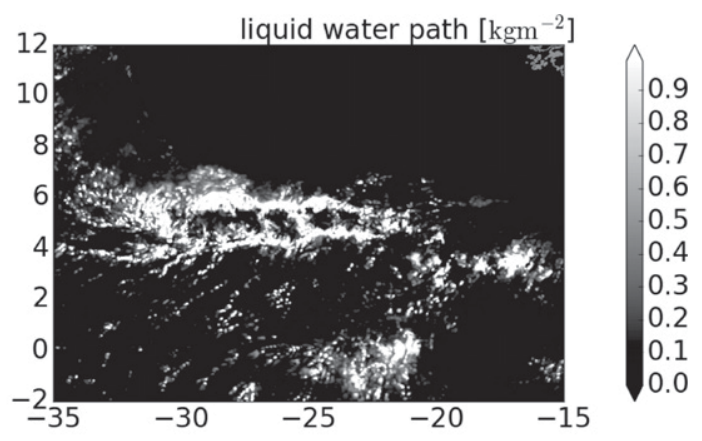

d) S-DCPL

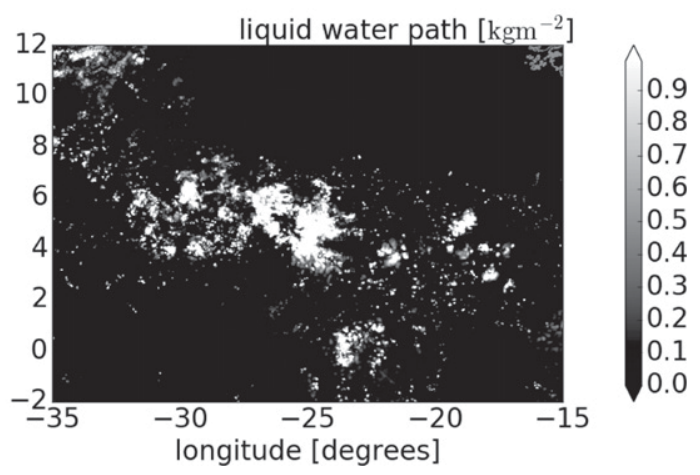

FIG. 15. A snapshot of the cloud liquid water path at 1200 UTC (20 Dec 2013) depicting the organization into (a),(b) cloud lines, (c) arcs, and (d) clusters. 


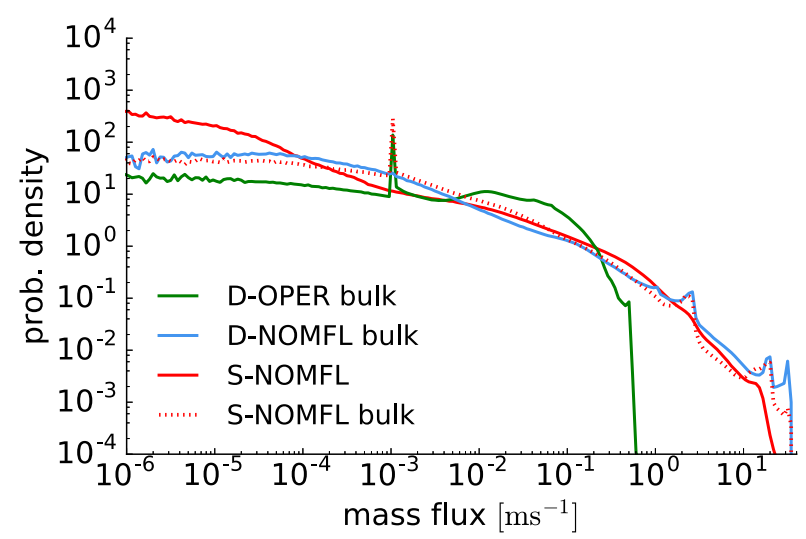

FIG. A1. Distribution of the convective mass flux in the three ICON experiments: D-OPER, D-NOMFL, and S-NOMFL. In all three cases, the closure of the T-B scheme provides a bulk value of the mass flux, denoted by "bulk." S-NOMFL is the only case here that produces the perturbed mass flux values in addition to the bulk values.

cloud lines that form a narrow precipitation band, while in S-NOMFL, deep clouds organize into arc structures visible in the plots of the liquid water path in Fig. 15. We show the liquid water path instead of the total water path or some other quantity because cold pools can be best identified at low levels as the liquid water clouds organize along their edges. These different organization modes result from the effects of subgrid convection without the mass flux limiters and including the stochastic effects that breaks the cloud lines apart into cloud clusters forming a wider precipitation band in S-DCPL (Fig. 15d). In addition, the resolved convective updrafts are stronger and deeper in S-NOMFL compared to S-DCPL because subgrid convection is active at places where deep resolved convection develops. Such stronger updrafts produce precipitation-induced cold pools that propagate meridionally and generate the cloud arcs and thereby increase the width of ITCZ (Fig. 15c) (previously suggested by Nolan et al. 2016). Such cloud arcs are only present in S-NOMFL where the resolved convection is locally strongly forced by the latent heat flux from the ocean's surface enhanced by the subgrid shallow convection. We cannot determine how realistic this development of cold pools within the ITCZ is by using only the present set of observations and analysis employed in our study, however based on the distribution of precipitation and how well it compares to observations, it is a plausible scenario.

Deepest clouds as observed by SEVIRI, depicted by the coldest brightness temperatures (BT) in the Fig. 11b, are not resolved in any of the experiments tested here. S-NOMFL produces a higher frequency of deep clouds in the higher range of BT, however, it fails to capture the frequency of the lower range of BT. It reproduces more deep clouds than the other cases because the parameterized stochastic shallow convection heats the subcloud layer and transports moisture out of the subcloud layer into the higher layers more effectively than the other cases and thereby supports development of deep clouds.

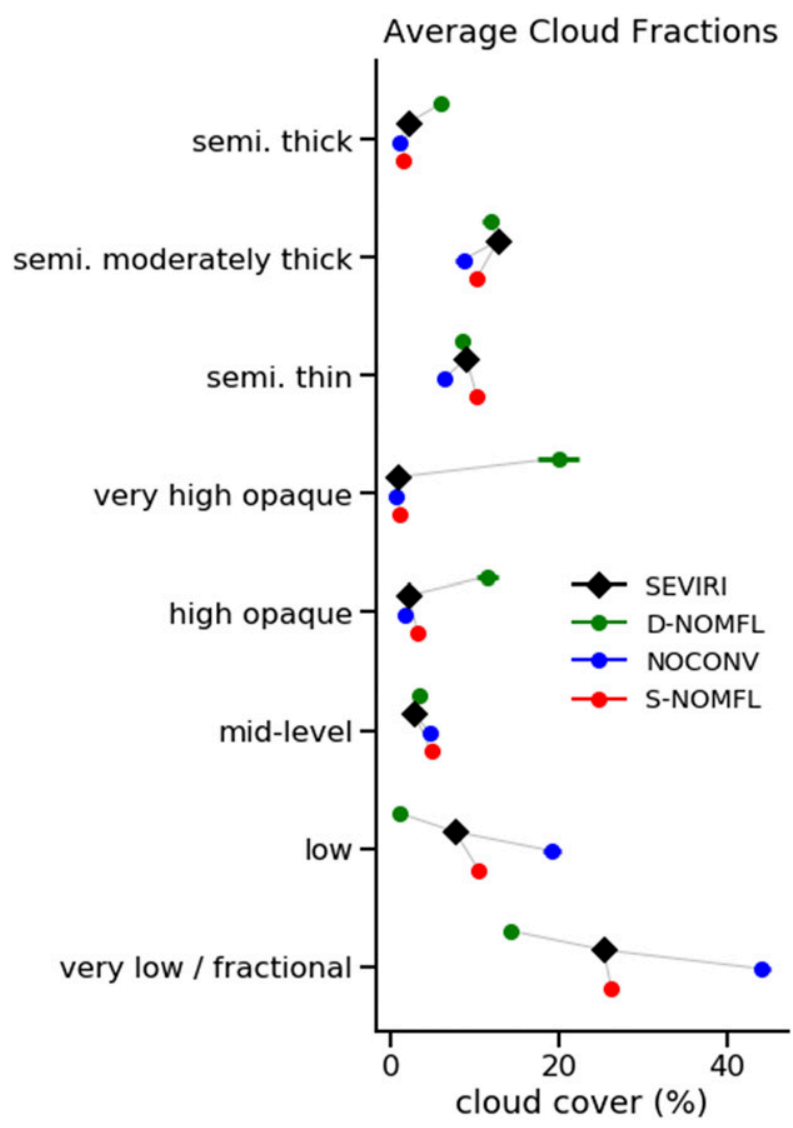

FIG. A2. Domain- and time-average cloud fraction of different cloud types. As in Fig. 4, but including D-NOMFL instead of D-OPER.

Due to the way the T-B convection scheme is formulated, shallow convection is active only in the initial stage of the deep cloud life cycle. In order for the deepest clouds to develop, such shallow convective transport in the subcloud layer would most probably be necessary during the entire cloud life cycle. To do that, a new mass flux closure and substantial changes to the convection scheme would need to be undertaken.

To isolate the impact of the stochastic element in the new shallow convection treatment, the underlying T-B scheme and closure have been kept as close to the operational ICON implementation as possible. The operational implementation of the T-B scheme in ICON was optimized for midlatitude continent, specifically for Germany, but is employed without modifications to the tropical Atlantic in our study. Thus, there is a potential for further improvements. Also, based on the sensitivity tests we conducted in the course of this study, we recognized the possibility of tuning of the S-NOMFL setup. The two parameters that have the most effect on the model performance are the mass flux limiters and $D_{\max }$. We found that the results in terms of the precipitation in the ITCZ are still not degraded when using the mass flux limit of $10 \mathrm{~kg} \mathrm{~m}^{-2} \mathrm{~s}^{-1}$, so higher numerical stability of the simulations can be achieved in the cases where the model runs are affected by a buildup of instability. 


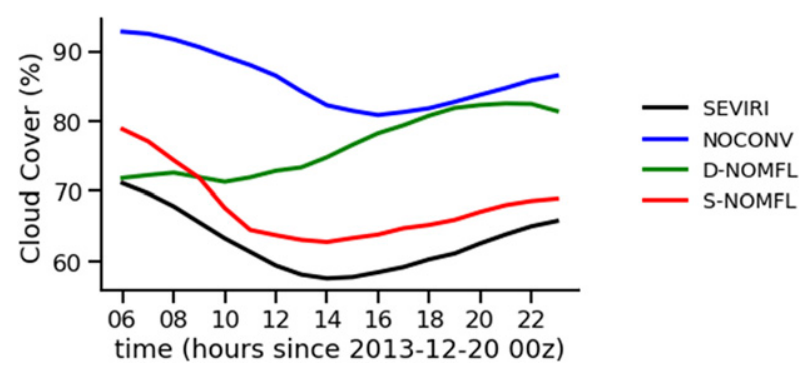

FIG. A3. Time series of the domain-average total cloud cover, as in Fig. 2a, but including D-NOMFL instead of D-OPER.

\section{Summary and conclusions}

A case study on 20 December 2013 was simulated using ICON in several different configurations to assess the local impact of stochastic shallow convection on resolved convection, clouds, and precipitation in the tropical Atlantic. The impact of stochastic shallow convection (S-NOMFL) was tested by comparing it to a case where no subgrid shallow convection scheme was included (NOCONV) and to a case that used the operational version of shallow convection without stochastic effects (D-OPER). In addition, the impact of the missing interaction between parameterized shallow convection and the resolved deep updrafts and deep clouds is tested in S-DCPL. Next to these four experiments, two additional experiments were conducted to isolate the local effects of the stochastic shallow convection in the ITCZ region and the trades by switching off the convective parameterization in the trades or the ITCZ region, respectively.

The most prominent result of our study is a decrease in the low-level cloud cover by approximately $20 \%$ on average due to the shallow convection parameterized via the stochastic scheme. The stochastic shallow convection increases the boundary layer height by more convective heating in the subcloud layer compared to the other experiments, and invigorates resolved convective circulations. Evaporation from the ocean's surface is enhanced and water is transported more effectively by stronger convective updrafts to higher atmospheric levels. The low-level cloud cover is in this case very close to the cloud cover observed by SEVIRI.

The stochastic parameterization of shallow convection invigorates the resolved convective circulations and widens the updraft region within the ITCZ. Moreover, we found that parameterized convection just beneath the resolved deep updrafts is responsible for stronger and deeper resolved updrafts, higher cloud tops and higher rain rates, which are responsible for a wider precipitation band in the ITCZ. Precipitation in the ITCZ is better simulated if the local shallow convective mixing is represented, independent of the shallow convective activity in the trades. The misrepresentation of the precipitation band in the NOCONV case is linked to the missing effects of the local shallow convection within the ITCZ. A remote interaction between shallow convection in the trades and the convection in the ITCZ was not possible in our experiments due to the brevity of the simulations. To confirm this hypotheses, we demonstrated that absent shallow convection in the trades does

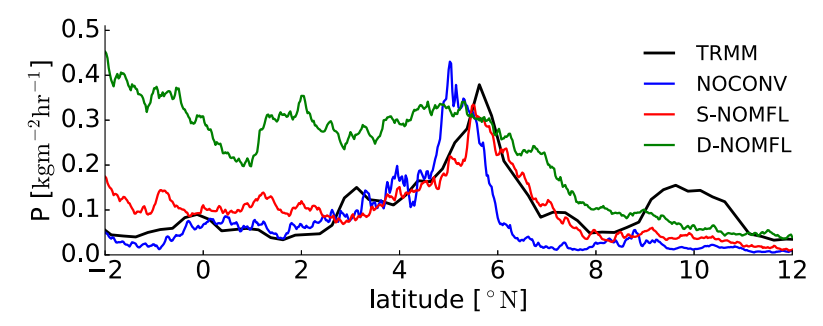

FIG. A4. Zonally averaged total daily precipitation.

not significantly affect the convection in the ITCZ, while the absence of shallow convection in the ITCZ has no significant effect on the flow in the trades in our experimental setup.

The change in the width of the precipitation band in the ITCZ is mainly related to the deactivation of the mass-flux limiters, which invigorates and spreads the updrafts in a wider region compared to the other cases, and by the stochastic effects that correct the mass flux distribution and limit the strength of updrafts in a physically constrained way (see section 1). Such physical constraints introduced through the stochastic scheme instead of the artificial mass flux limiters ensure that the resolved deep convection is strong enough to promote the organization of deep clouds into arc structures. The underlying mechanism is most likely the propagation of the cold pools that result from the evaporative cooling of convective precipitation. The propagation of the arc-shaped squall lines in the meridional direction happens in the case of the active shallow convection parameterization beneath resolved deep updrafts (S-NOMFL), while in the case where subgrid shallow convection is decoupled from the resolved deep convection, such arc structures and propagation do not occur. It is, however, not clear why the cold pools in the strongly organized NOCONV case do not propagate in the same manner to create cloud arcs but clouds remain organized in straight lines.

We demonstrated in this paper, how the effective forcing of the atmosphere by the ocean is achieved by the means of a parameterization of shallow convection as the most efficient transport mechanism in the BL. In summary, missing local effects of the subgrid shallow convection scheme result in a too-high low-level cloud cover, a too-weak resolved circulations, insufficient deep clouds, and a too-narrow precipitation band in the ITCZ. Even an imperfect parameterization of shallow convection with known deficiencies at the CP resolutions, as used in the present study, results in an impact that combined with a potential remote impact on longer time scales could be a crucial component of every weather and climate modeling effort at convection-permitting scales.

Acknowledgments. This research was carried out in the Hans Ertel Center for Weather Research (HErZ). This German research network of universities, research institutions, and DWD is funded by the BMVI (Federal Ministry of Transport and Digital Infrastructure). The simulations were conducted on the super computer system of the German Climate Computing Center (DKRZ). Fabian Senf acknowledge funding within the High Definition Clouds and Precipitation 

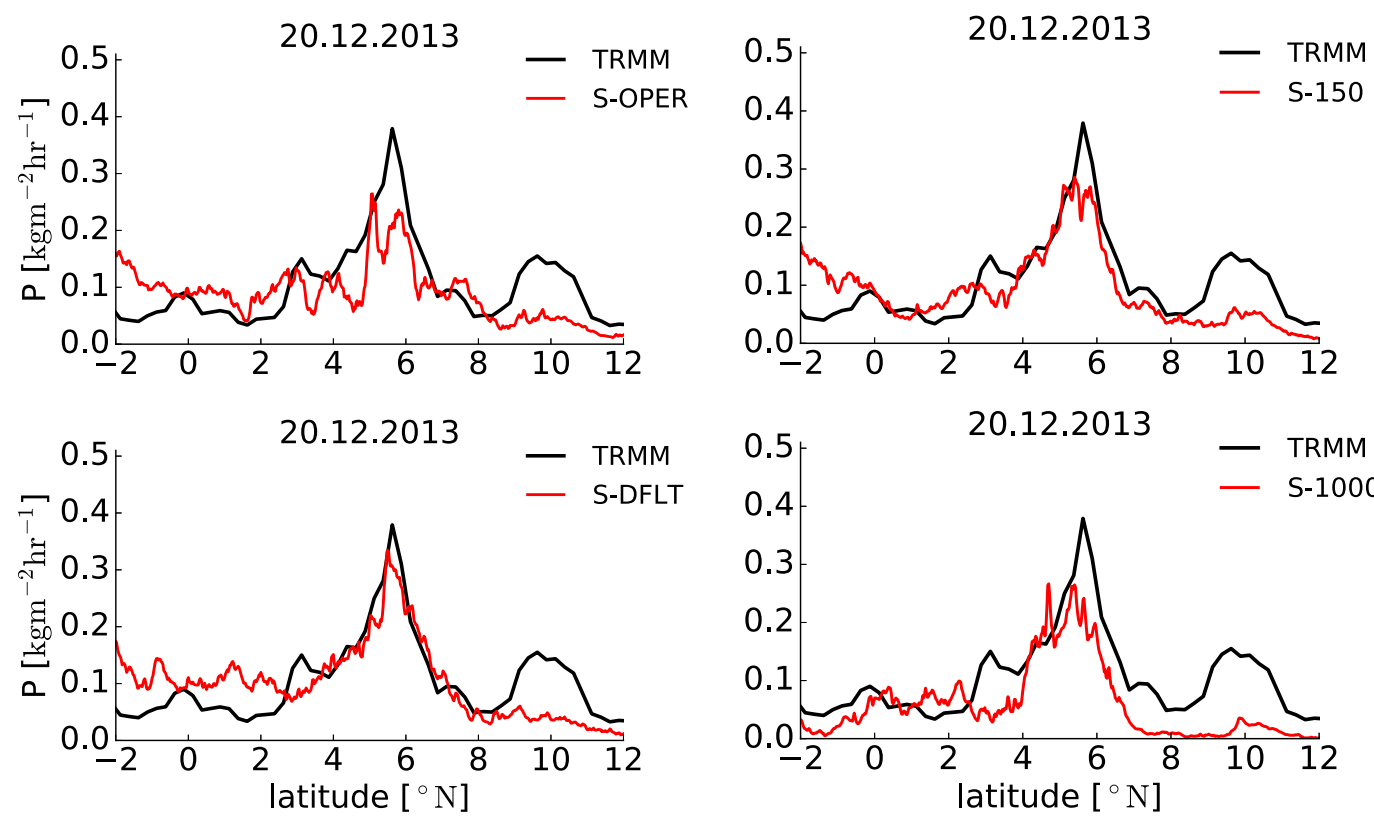

FIG. B1. The average precipitation rate in the four experiments that examine the sensitivity to $D_{\max }$ compared to TRMM.

for advancing Climate Prediction $\left[\mathrm{HD}(\mathrm{CP})^{2}\right]$ project funded by the BMBF (German Ministry for Education and Research) under respective Grants 01 LK1507C and 01 LK1503F. We also thank EUMETSAT for producing the SEVIRI data, which have been obtained from the TROPOS satellite data archive. Special thanks to Takuya Jinno for his analysis of several preliminary experiments not included in the final version. We also thank Christian Jakob, Peter Bechtold, and Cathy Hohenegger for their suggestions and most useful discussions. Thanks to Julia Windmiller for discussions about cold pools and for providing comments on an early version of the manuscript. We are also grateful to the two anonymous reviewers for the most helpful comments and suggestions.

Data availability statement. Primary data and scripts used in the analysis and other supplementary information that may be useful in reproducing the author's work are archived by the Max Planck Institute for Meteorology and can be obtained at http://hdl.handle.net/21.11116/0000-0006-0297-D and https://cera-www.dkrz.de/WDCC/ui/cerasearch/entry? acronym=DKRZ $\_L T A \backslash 731 \_d s 00001$. Jupyter notebooks for the analysis of brightness temperatures and cloud-type statistics can be download under http://doi.org/10.5281/zenodo.3952525.

\section{APPENDIX A}

\section{Effects of Stochastic Sampling}

The improvement of the modeled clouds and precipitation that comes from deactivating the limiters of the parameterized mass flux would not be possible without the stochastic scheme. The quasi-random sampling of the cloud-base mass flux corrects the spatial and temporal distribution of the mass flux and thereby corrects the distribution of clouds and precipitation. To isolate the effects of the stochastic cloud sampling from the effects of imposing the mass-flux limits, here we include an additional simulation using the configuration of the T-B scheme without the stochastic scheme and with effectively disabled mass flux limiters (D-NOMFL). In this way we directly compare the S-NOMFL case with the deterministic T-B that includes no limiters in the convective mass flux and keeps the default values of the tuning parameters (D-NOMFL), so that the only difference between the two is in the stochastic sampling and other features of the stochastic scheme.

The stochastic sampling changes the mass flux distribution in three significant ways (Fig. A1). First, it greatly increases the probability of occurrence of very low values of mass flux, because of the long-tailed shape of the distribution used for the mass flux sampling (a mixed Weibull distribution with two modes). This increase in low value probability enables more coherent behavior of the convection scheme and eliminates intermittent (on-off) behavior. Second, the stochastic sampling lightens the tail of the distribution, by reducing the probability of very high, unrealistic mass flux values to a very low number of points (Fig. A1). This makes the stochastic version more stable when the mass flux limiters are disabled. And third, it smooths out the abrupt artificial peaks in the mass flux distribution that are present in the deterministic versions due to the way the convection scheme is constructed. We did not attempt to fix the underlying convection scheme to avoid such peaks, but we tried to keep the scheme as similar as possible to the D-OPER code version.

The contrast between the mass flux distributions with and without limiters in the operational model demonstrates that the limiters do not act occasionally to catch a few outliers, but routinely adjust the calculated mass flux values of the T-B 


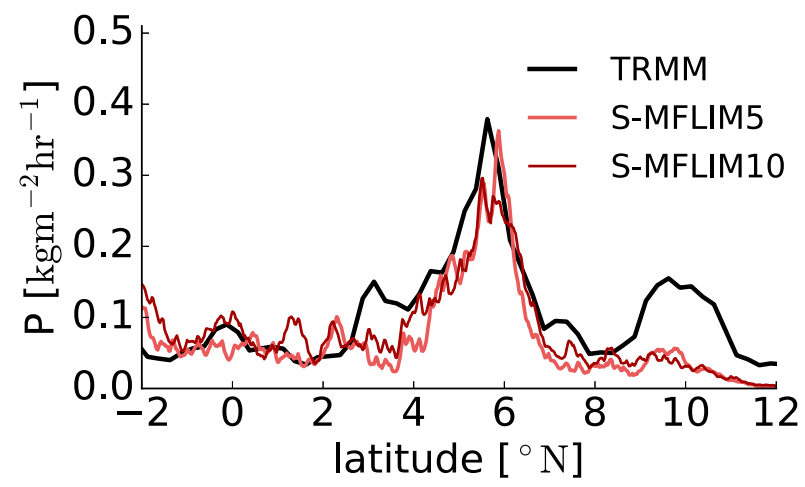

FIG. B2. The average precipitation rate in the two experiments that test the sensitivity to the mass flux limiters compared to TRMM.

scheme and are thus an integral part of that scheme. Without the limiters, the model produces such an unrealistic cloud state that it is no longer fit for operational use. We show this unrealistic cloud state of D-NOMFL in Figs. A2 and A3. The average cloud fraction of low and very low clouds is too low, while the cloud fraction of the high opaque clouds is unrealistically high in D-NOMFL (Fig. A2). In this case, the total cloud cover increases during the day, which is a trend opposite of the observed one (Fig. A3). Such a high cloud fraction of the high opaque clouds in D-NOMFL produces a too-broad precipitation band in the ITCZ (Fig. A4).

\section{APPENDIX B}

\section{Sensitivity Tests}

We conducted several additional experiments to document the sensitivity tests done to accompany the analysis of the ICON model performance in the case study of 20 December 2013 over the tropical Atlantic. The additional experiments are based on the stochastic version of the $\mathrm{T}-\mathrm{B}$ shallow convection, where the mass flux limiters are inactive, including

1) configurations used for the sensitivity tests of the three additional values of $D_{\max }$, NWP tuning value of around $77 \mathrm{hPa}$ (S-OPER), $150 \mathrm{hPa}$ (S-150), and $1000 \mathrm{hPa}$ (S1000), and

2) configurations used for the sensitivity tests of the two additional values of the mass flux hard limit, S-MFLIM5 and S-MFLIM10.

We did not attempt to tune the model to match the single main case of the current study better, but in principle the performance of the stochastic shallow convection can be tuned. The two most sensitive parameters are $D_{\max }$ and the mass flux limiters and thus it would be most effective to tune these parameters.

\section{a. Sensitivity to the maximum allowed shallow cloud depth}

The maximum allowed shallow cloud depth $D_{\max }$ has the most effects on the simulation results (Fig. B1). The activity of shallow convection in the domain decreases with the increase of $D_{\max }$ from about 77 to $1000 \mathrm{hPa}$ (not shown), making the shallow-convecting regions represented more correctly from about 150 to $200 \mathrm{hPa}$ and then again less correctly up to $1000 \mathrm{hPa}$. The distribution of precipitation rates is highly affected by the choice of $D_{\max }$. When $D_{\max }$ is set to about $77 \mathrm{hPa}$, which is a resolution-dependent operational tuning, the precipitation band in the ITCZ region becomes very narrow and noisier compared to our default S-NOMFL case. The case with $D_{\max }$ set to $150 \mathrm{hPa}$ improves the representation of precipitation in the ITCZ, while S-NOMFL matches the TRMM almost perfectly (Fig. B1). In the case of $D_{\text {max }}=1000 \mathrm{hPa}$ the precipitation band is shifted to the south and the similarity between the modeled and observed signatures is reduced (Fig. B1).

\section{b. Sensitivity to a mass-flux limit}

In principle, decreasing the value of a mass flux limiter from 100 to 10 or $5 \mathrm{~kg} \mathrm{~m}^{-2} \mathrm{~s}^{-1}$ affects the simulated case in a similar way as the decrease in $D_{\max }$ from its default value of $200 \mathrm{hPa}$ affects it. The convective intensity decreases on average as the probability of small range of the mass-flux values increases, while spatial area of active shallow convection increases as a result (not shown). The average precipitation rate in the ITCZ shows less similarity to TRMM compared to S-NOMFL in the case of the mass-flux limiter set to $10 \mathrm{~kg} \mathrm{~m}^{-2} \mathrm{~s}^{-1}$, while in the case of the mass flux limiter set to $5 \mathrm{~kg} \mathrm{~m}^{-2} \mathrm{~s}^{-1}$ it is further slightly degraded (Fig. B2). However, depending on the application, both test values of the mass flux limiter might still be satisfying.

\section{REFERENCES}

Baldauf, M., A. Seifert, J. Förstner, D. Majewski, M. Raschendorfer, and T. Reinhardt, 2011: Operational convective-scale numerical weather prediction with the COSMO model: Description and sensitivities. Mon. Wea. Rev., 139, 3887-3905, https://doi.org/ 10.1175/MWR-D-10-05013.1.

Bechtold, P., M. Köhler, T. Jung, F. Doblas-Reyes, M. Leutbecher, M. J. Rodwell, F. Vitart, and G. Balsamo, 2008: Advances in simulating atmospheric variability with the ECMWF model: From synoptic to decadal time-scales. Quart. J. Roy. Meteor. Soc., 134, 1337-1351, https://doi.org/10.1002/qj.289.

Behrangi, A., M. Lebsock, S. Wong, and B. Lambrigtsen, 2012: On the quantification of oceanic rainfall using spaceborne sensors. J. Geophys. Res., 117, D20105, https://doi.org/ 10.1029/2012JD017979.

Brisson, E., K. Van Weverberg, M. Demuzere, A. Devis, S. Saeed, M. Stengel, and N. P. M. van Lipzig, 2016: How well can a convection-permitting climate model reproduce decadal statistics of precipitation, temperature and cloud characteristics? Climate Dyn., 47, 3043-3061, https://doi.org/10.1007/s00382-016-3012-z.

Bryan, G. H., J. C. Wyngaard, and J. M. Fritsch, 2003: Resolution requirements for the simulation of deep moist convection. Mon. Wea. Rev., 131, 2394-2416, https://doi.org/10.1175/15200493(2003)131<2394:RRFTSO>2.0.CO;2.

Ching, J., R. Rotunno, M. LeMone, A. Martilli, B. Kosovic, P. A. Jimenez, and J. Dudhia, 2014: Convectively induced secondary circulations in fine-grid mesoscale numerical weather prediction models. Mon. Wea. Rev., 142, 3284-3302, https:// doi.org/10.1175/MWR-D-13-00318.1.

Craig, G. C., and B. G. Cohen, 2006: Fluctuations in an equilibrium convective ensemble, Part I: Theoretical formulation. J. Atmos. Sci., 63, 1996-2004, https://doi.org/10.1175/JAS3709.1. 
Derrien, M., and H. Le Gléau, 2005: MSG/SEVIRI cloud mask and type from SAFNWC. Int. J. Remote Sens., 26, 4707-4732, https://doi.org/10.1080/01431160500166128.

Fosser, G., S. Khodayar, and P. Berg, 2015: Benefit of convection permitting climate model simulations in the representation of convective precipitation. Climate Dyn., 44, 45-60, https:// doi.org/10.1007/s00382-014-2242-1.

Fu, Q., 1996: An accurate parameterization of the solar radiative properties of cirrus clouds for climate models. J. Climate, $\mathbf{9}$, 2058-2082, https://doi.org/10.1175/1520-0442(1996)009<2058: AAPOTS $>2.0 . \mathrm{CO} ; 2$.

Heinze, R., and Coauthors, 2017: Large-eddy simulations over Germany using ICON: A comprehensive evaluation. Quart. J. Roy. Meteor. Soc., 143, 69-100, https://doi.org/10.1002/ qj.2947.

Heise, E., B. Ritter, and E. Schrodin, 2006: Operational implementation of the multilayer soil model TERRA. Tech. Rep., 20 pp.

Hentgen, L., N. Ban, N. Kröner, D. Leutwyler, and C. Schär, 2019: Clouds in convection-resolving climate simulations over Europe. J. Geophys. Res. Atmos., 124, 3849-3870, https:// doi.org/10.1029/2018JD030150.

Hohenegger, C., L. Kornblueh, D. Klocke, T. Becker, G. Cioni, J. F. Engels, U. Schulzweida, and B. Stevens, 2020: Climate statistics in global simulations of the atmosphere, from 80 to $2.5 \mathrm{~km}$ grid spacing. J. Meteor. Soc. Japan, 98, 73-91, https:// doi.org/10.2151/jmsj.2020-005.

Johnson, R. H., T. M. Rickenbach, S. A. Rutledge, P. E. Ciesielski, and W. H. Schubert, 1999: Trimodal characteristics of tropical convection. J. Climate, 12, 2397-2418, https://doi.org/10.1175/ 1520-0442(1999)012<2397:TCOTC>2.0.CO;2.

Keil, C., A. Tafferner, and T. Reinhardt, 2006: Synthetic satellite imagery in the Lokal-Modell. Atmos. Res., 82, 19-25, https:// doi.org/10.1016/j.atmosres.2005.01.008.

Klocke, D., M. Brueck, C. Hohenegger, and B. Stevens, 2017: Rediscovery of the doldrums in storm-resolving simulations over the tropical Atlantic. Nat. Geosci., 10, 891-896, https:// doi.org/10.1038/s41561-017-0005-4.

Langhans, W., J. Schmidli, O. Fuhrer, S. Bieri, and C. Schär, 2013: Long-term simulations of thermally driven flows and orographic convection at convection-parameterizing and cloud-resolving resolutions. J. Appl. Meteor. Climatol., 52, 1490-1510, https://doi.org/10.1175/JAMC-D-12-0167.1.

Liu, C., and Coauthors, 2017: Continental-scale convectionpermitting modeling of the current and future climate of North America. Climate Dyn., 49, 71-95, https://doi.org/ 10.1007/s00382-016-3327-9.

Martin, G. M., D. W. Johnson, and A. Spice, 1994: The measurement and parameterization of effective radius of droplets in warm stratocumulus clouds. J. Atmos. Sci., 51, 1823-1842, https://doi.org/10.1175/1520-0469(1994)051<1823: TMAPOE $>2.0 . \mathrm{CO} ; 2$.

Matricardi, M., F. Chevallier, G. Kelly, and J.-N. Thépaut, 2004: An improved general fast radiative transfer model for the assimilation of radiance observations. Quart. J. Roy. Meteor. Soc., 130, 153-173, https://doi.org/10.1256/qj.02.181.

McFarquhar, G. M., S. Iacobellis, and R. C. J. Somerville, 2003: SCM simulations of tropical ice clouds using observationally based parameterizations of microphysics. J. Climate, 16, 1643-1664, https://doi.org/10.1175/1520-0442(2003)016<1643: SSOTIC $>2.0 . \mathrm{CO} ; 2$.

Mlawer, E. J., S. J. Taubman, P. D. Brown, M. J. Iacono, and S. A. Clough, 1997: Radiative transfer for inhomogeneous atmospheres: RRTM, a validated correlated-k model for the longwave. J. Geophys. Res., 102, 16 663-16 682, https:// doi.org/10.1029/97JD00237.

Nam, C., S. Bony, J.-L. Dufresne, and H. Chepfer, 2012: The 'too few, too bright' tropical low-cloud problem in CMIP5 models. Geophys. Res. Lett., 39, L21801, https://doi.org/ 10.1029/2012GL053421.

Neggers, R., B. Stevens, and J. D. Neelin, 2006: A simple equilibrium model for shallow-cumulus-topped mixed layers. Theor. Comput. Fluid Dyn., 20, 305-322, https://doi.org/10.1007/ s00162-006-0030-1.

Nolan, D. S., S. N. Tulich, and J. E. Blanco, 2016: ITCZ structure as determined by parameterized versus explicit convection in aquachannel and aquapatch simulations. J. Adv. Model. Earth Syst., 8, 425-452, https://doi.org/10.1002/2015MS000560.

Pedruzo-Bagazgoitia, X., P. A. Jiménez, J. Dudhia, and J. VilàGuerau de Arellano, 2019: Shallow cumulus representation and its interaction with radiation and surface at the convection gray zone. Mon. Wea. Rev., 147, 2467-2483, https://doi.org/ 10.1175/MWR-D-19-0030.1.

Petch, J. C., A. R. Brown, and M. E. B. Gray, 2002: The impact of horizontal resolution on the simulations of convective development over land. Quart. J. Roy. Meteor. Soc., 128, 2031-2044, https://doi.org/10.1256/003590002320603511.

Prein, A. F., and Coauthors, 2015: A review on regional convectionpermitting climate modeling: Demonstrations, prospects, and challenges. Rev. Geophys., 53, 323-361, https://doi.org/10.1002/ 2014RG000475.

Pscheidt, I., F. Senf, R. Heinze, H. Deneke, S. Trömel, and C. Hohenegger, 2019: How organized is deep convection over Germany? Quart. J. Roy. Meteor. Soc., 145, 2366-2384, https:// doi.org/10.1002/qj.3552.

Raschendorfer, M., 2001: The new turbulence parameterization of LM. COSMO Newsletter, No. 1, Consortium for Small-Scale Modeling, Offenbach, Germany, 89-97, http://www.cosmo-model.org/content/model/documentation/ newsLetters/newsLetter01/newsLetter_01.pdf.

Reinhardt, T., and A. Seifert, 2006: A three-category ice scheme for the LMK. COSMO Newsletter, No. 6, Consortium for Small-Scale Modeling, Offenbach, Germany, 115-120.

Roberts, N. M., and H. W. Lean, 2008: Scale-selective verification of rainfall accumulations from high-resolution forecasts of convective events. Mon. Wea. Rev., 136, 78-97, https://doi.org/ 10.1175/2007MWR2123.1.

Rossow, W. B., and R. A. Schiffer, 1999: Advances in understanding clouds from ISCCP. Bull. Amer. Meteor. Soc., 80, 2261-2287, https://doi.org/10.1175/1520-0477(1999)080<2261: AIUCFI $>2.0 . \mathrm{CO} ; 2$.

Sakradzija, M., and C. Hohenegger, 2017: What determines the distribution of shallow convective mass flux through a cloud base? J. Atmos. Sci., 74, 2615-2632, https://doi.org/10.1175/ JAS-D-16-0326.1.

, and D. Klocke, 2018: Physically constrained stochastic shallow convection in realistic kilometer-scale simulations. $J$. Adv. Model. Earth Syst., 10, 2755-2776, https://doi.org/10.1029/ 2018MS001358.

— A. Seifert, and T. Heus, 2015: Fluctuations in a quasi-stationary shallow cumulus cloud ensemble. Nonlinear Processes Geophys., 22, 65-85, https://doi.org/10.5194/npg-22-65-2015.

$\longrightarrow,-$, and A. Dipankar, 2016: A stochastic scale-aware parameterization of shallow cumulus convection across the convective gray zone. J. Adv. Model. Earth Syst., 8, 786-812, https://doi.org/10.1002/2016MS000634. 
Saunders, R., M. Matricardi, and P. Brunel, 1999: An improved fast radiative transfer model for assimilation of satellite radiance observations. Quart. J. Roy. Meteor. Soc., 125, 1407-1425, https://doi.org/10.1002/qj.1999.49712555615.

Scheck, L., P. Frèrebeau, R. Buras-Schnell, and B. Mayer, 2016: A fast radiative transfer method for the simulation of visible satellite imagery. J. Quant. Spectrosc. Radiat. Transfer, 175, 54-67, https://doi.org/10.1016/j.jqsrt.2016.02.008.

—_, M. Weissmann, and B. Mayer, 2018: Efficient methods to account for cloud-top inclination and cloud overlap in synthetic visible satellite images. J. Atmos. Oceanic Technol., 35, 665-685, https://doi.org/10.1175/JTECH-D-17-0057.1.

Schmetz, J., P. Pili, S. Tjemkes, D. Just, J. Kerkmann, S. Rota, and A. Ratier, 2002: An introduction to Meteosat Second Generation (MSG). Bull. Amer. Meteor. Soc., 83, 977-992, https://doi.org/10.1175/BAMS-83-7-Schmetz-2.

Seifert, A., 2008: A revised cloud microphysical parameterization for COSMO-LME. COSMO Newsletter, No. 7, Consortium for Small-Scale Modeling, Offenbach, Germany, 25-28.

Senf, F., and H. Deneke, 2017: Uncertainties in synthetic Meteosat SEVIRI infrared brightness temperatures in the presence of cirrus clouds and implications for evaluation of cloud microphysics. Atmos. Res., 183, 113-129, https://doi.org/10.1016/ j.atmosres.2016.08.012.

—, D. Klocke, and M. Brueck, 2018: Size-resolved evaluation of simulated deep tropical convection. Mon. Wea. Rev., 146, 2161-2182, https://doi.org/10.1175/MWR-D-17-0378.1.

—, A. Voigt, N. Clerbaux, A. Hünerbein, and H. Deneke, 2020: Increasing resolution and resolving convection improves the simulation of cloud-radiative effects over the North Atlantic.
J. Geophys. Res. Atmos., 125, e2020JD032667, https://doi.org/ 10.1029/2020JD032667.

Slingo, J., and Coauthors, 1994: Mean climate and transience in the tropics of the UGAMP GCM: Sensitivity to convective parametrization. Quart. J. Roy. Meteor. Soc., 120, 881-922, https://doi.org/10.1002/qj.49712051807.

Stevens, B., and Coauthors, 2019: A high-altitude long-range aircraft configured as a cloud observatory: The NARVAL expeditions. Bull. Amer. Meteor. Soc., 100, 1061-1077, https:// doi.org/10.1175/BAMS-D-18-0198.1.

Stull, R. B., 1985: A fair-weather cumulus cloud classification scheme for mixed-layer studies. J. Climate Appl. Meteor., 24, 49-56, https://doi.org/10.1175/1520-0450(1985)024<0049: $\mathrm{AFWCCC}>2.0 . \mathrm{CO} ; 2$.

Tiedtke, M., 1989: A comprehensive mass flux scheme for cumulus parameterization in large-scale models. Mon. Wea. Rev., 117, 1779-1800, https://doi.org/10.1175/1520-0493(1989)117<1779: ACMFSF $>2.0 . \mathrm{CO} ; 2$.

— W. Heckley, and J. Slingo, 1988: Tropical forecasting at ECMWF: The influence of physical parametrization on the mean structure of forecasts and analyses. Quart. J. Roy. Meteor. Soc., 114, 639-664, https://doi.org/10.1002/qj.49711448106.

TRMM, 2011: TRMM (TMPA) rainfall estimate L3 3 hour 0.25 degree $\times 0.25$ degree V7. Accessed 27 August 2019, https:// doi.org/10.5067/TRMM/TMPA/3H/7.

Zängl, G., D. Reinert, P. Rípodas, and M. Baldauf, 2015: The ICON (ICOsahedral Non-hydrostatic) modelling framework of DWD and MPI-M: Description of the non-hydrostatic dynamical core. Quart. J. Roy. Meteor. Soc., 141, 563-579, https:// doi.org/10.1002/qj.2378. 